\title{
Quasi-polynomial mixing of the 2D stochastic Ising model with "plus" boundary up to criticality
}

Received December 21, 2010 and in revised form May 4, 2011

\begin{abstract}
We considerably improve upon the recent result of [37] on the mixing time of Glauber dynamics for the 2D Ising model in a box of side $L$ at low temperature and with random boundary conditions whose distribution $\mathbf{P}$ stochastically dominates the extremal plus phase. An important special case is when $\mathbf{P}$ is concentrated on the homogeneous all-plus configuration, where the mixing time $T_{\mathrm{MIX}}$ is conjectured to be polynomial in $L$. In [37] it was shown that for a large enough inverse temperature $\beta$ and any $\varepsilon>0$ there exists $c=c(\beta, \varepsilon)$ such that $\lim _{L \rightarrow \infty} \mathbf{P}\left(T_{\mathrm{MIX}} \geq \exp \left(c L^{\varepsilon}\right)\right)=0$. In particular, for the all-plus boundary conditions and $\beta$ large enough, $T_{\mathrm{MIX}} \leq \exp \left(c L^{\varepsilon}\right)$.

Here we show that the same conclusions hold for all $\beta$ larger than the critical value $\beta_{c}$ and with $\exp \left(c L^{\varepsilon}\right)$ replaced by $L^{c \log L}$ (i.e. quasi-polynomial mixing). The key point is a modification of the inductive scheme of [37] together with refined equilibrium estimates that hold up to criticality, obtained via duality and random-line representation tools for the Ising model. In particular, we establish new precise bounds on the law of Peierls contours which complement the Brownian bridge picture established e.g. in [20,22,23].
\end{abstract}

Keywords. Ising model, mixing time, phase coexistence, Glauber dynamics

\section{Introduction}

The Ising model on lattices at and near criticality has been the focus of numerous research papers since its introduction in 1925, establishing it as one of the most studied models in mathematical physics. In two dimensions the model was exactly solved by Onsager [40] in 1944, determining its critical inverse temperature $\beta_{c}=\frac{1}{2} \log (1+\sqrt{2})$ in the absence of an external magnetic field. While the classical study of the Ising model concentrated on its static properties, over the last three decades significant efforts were dedicated to the analysis of stochastic dynamical systems that both model its evolution and provide

E. Lubetzky: Microsoft Research, One Microsoft Way, Redmond, WA 98052-6399, USA; e-mail: eyal@microsoft.com

F. Martinelli: Dipartimento di Matematica, Università Roma Tre,

Largo S. Murialdo 1, 00146 Roma, Italia; e-mail: martin@mat.uniroma3.it

A. Sly: Microsoft Research, One Microsoft Way, Redmond, WA 98052-6399, USA;

e-mail: allansly@microsoft.com

F. L. Toninelli: CNRS and ENS Lyon, Laboratoire de Physique,

46 Allée d'Italie, 69364 Lyon, France; e-mail: fabio-lucio.toninelli@ens-lyon.fr

Mathematics Subject Classification (2010): 60K35, 82C20 
efficient methods of sampling it. Of particular interest is the interplay between the behaviors of the static and dynamical models as they both undergo a phase transition at the critical $\beta_{c}$.

The Glauber dynamics for the Ising model (also known as the stochastic Ising model), introduced by Glauber [19] in 1963, is considered to be the most natural sampling method for it, with notable examples including heat-bath and Metropolis. It is known that on a box of side-length $L$ in $\mathbb{Z}^{2}$ with free boundary conditions (b.c.), alongside the phase transition in the range of spin-spin correlations in the static Ising model around $\beta_{c}$, the corresponding Glauber dynamics exhibits a critical slowdown: Its mixing time (formally defined in \$1.1) transitions from being logarithmic in $L$ in the high temperature regime $\beta<\beta_{c}$ to being exponentially large in $L$ in the low temperature regime $\beta>\beta_{c}$, en route following a power law at the critical $\beta_{c}$.

One of the most fundamental open problems in the study of the stochastic Ising model is understanding the system's behavior in the so-called phase-coexistence region under homogeneous boundary conditions, e.g. all-plus boundary. In the presence of these b.c. the (-) phase becomes unstable and as such the reduced bottleneck between the two phases drastically accelerates the rate of convergence of the dynamics to equilibrium. Indeed, in this case the Glauber dynamics is known to mix in time that is subexponential in the surface area of the box, in contrast to its low-temperature behavior with free boundary. The central and longstanding conjecture addressing this phenomenon states that the mixing time of Glauber dynamics for the Ising model on a box of side-length $L$ with all-plus boundary conditions is at most polynomial in $L$ at any temperature.

So far this has been confirmed on the 2D lattice throughout the one-phase region $\beta<\beta_{c}$ (see [33,34]) and very recently at the critical $\beta=\beta_{c}$ (see [30]). Despite intensive efforts over the last two decades, establishing a power-law behavior for the mixing of Glauber dynamics at the phase-coexistence region $\beta>\beta_{c}$ under the all-plus b.c. remains an enticing open problem.

In [15] the precise order of mixing in this regime on a 2D square lattice of side-length $L$ was conjectured to be $L^{2}$ in accordance with Lifshitz's law (see [28] and also [12,39, 47]). The heuristic behind this prediction argues that when a droplet of the $(-)$ phase is surrounded by the $(+)$ phase at low temperature it proceeds to shrink according to the mean-curvature of the interface between them. Unfortunately, rigorous analysis is still quite far from establishing the expected Lifshitz behavior of $O\left(L^{2}\right)$ mixing.

Until recently the best upper bound on the mixing at the phase-coexistence region under the all-plus boundary was $\exp \left(L^{1 / 2+o(1)}\right)$ due to [32] and valid for large enough $\beta$. This bound from 1994 was substantially improved in a recent breakthrough paper [37], where it was shown (as a special case of a result on a wider class of b.c.) that for a sufficiently large $\beta$ and any $\varepsilon>0$ the mixing time is $\exp \left(O\left(L^{\varepsilon}\right)\right)$. The approach of [37] hinged on a novel inductive scheme on boxes with random boundary conditions, combined with a careful use of the so-called Peres-Winkler censoring inequality; these ideas form the foundation of the present paper. Note that the requirement of large $\beta$ in $[32,37]$ was essential in order to make use of results of [14] on the Wulff construction, available only at low enough temperature by cluster expansion methods. For smaller values of 
$\beta>\beta_{c}$ the best known estimates on the mixing time are due to [11] and of the weaker form $\exp (o(L))$.

In this work we improve these estimates into an upper bound of the form $L^{O(\log L)}$ on the mixing-time (i.e. quasi-polynomial in the side-length $L$ ) valid for any $\beta>\beta_{c}$. The key to our analysis is a modification of the recursive framework introduced in [37] combined with refined equilibrium estimates that hold up to criticality. To establish these, in lieu of relying on cluster-expansions, we utilize duality and the random-line representation machinery for the high temperature Ising model developed in [43,44] (see also the recent developments in $[7,8]$ and the discussion at the beginning of $\$ 5$ ).

A key new element of our proof concerns fine estimates on the fluctuations of cluster boundaries. Whenever the boundary is pinned at two vertices $u=(0,0)$ and $v=(\ell, 0)$, the contour of the cluster is known to converge to the Brownian bridge (cf. [13, 22, 23]). This does not, however, allow us to directly estimate the probability of events when these converge to 0 in the limit. In particular, we are interested in: (i) a Gaussian bound for the probability that the contour would reach height $x \sqrt{\ell}$ (established in Theorem 5.3); (ii) the probability that the contour remains in the upper half-plane, an event that would have probability $1 / \ell$ were the contour to behave like a $1 \mathrm{D}$ random walk of length $\ell$ conditioned to return to 0 . In $\S 5$ (see Theorem 5.1) we prove that up to multiplicative constants this indeed holds for a given contour.

These then provide important tools in estimating the probability of various other events characterizing the Ising interfaces at equilibrium.

\subsection{Glauber dynamics for the Ising model}

Let $\Lambda$ be a generic finite subset of $\mathbb{Z}^{2}$. Write $x \sim y$ for the nearest neighbor relation in $\mathbb{Z}^{2}$ (i.e. $x \sim y$ if $\max _{i=1,2}\left|x_{i}-y_{i}\right|=1$ ) and define $\partial \Lambda$, the boundary of $\Lambda$, to be the nearest neighbors of $\Lambda$ in $\mathbb{Z}^{2} \backslash \Lambda$ :

$$
\partial \Lambda:=\left\{x \in \mathbb{Z}^{2} \backslash \Lambda: x \sim y \text { for some } y \in \Lambda\right\} .
$$

The classical Ising model on $\Lambda$ with no external magnetic field is a spin-system whose set of possible configurations is $\Omega_{\Lambda}=\{-1,+1\}^{\Lambda}$. Each configuration $\sigma \in \Omega_{\Lambda}$ corresponds to an assignment of plus/minus spins to the sites in $\Lambda$ and has a statistical weight determined by the Hamiltonian

$$
H_{\Lambda}^{\tau}(\sigma)=-\sum_{\substack{x, y \in \Lambda \\ x \sim y}} \sigma_{x} \sigma_{y}-\sum_{\substack{x \in \Lambda, y \in \partial \Lambda \\ x \sim y}} \sigma_{x} \tau_{y}
$$

where $\tau \in \Omega_{\partial \Lambda}$ forms the boundary conditions (b.c.) of the system. The Gibbs measure associated to the spin-system with boundary conditions $\tau$ is

$$
\pi_{\Lambda}^{\tau}(\sigma)=\frac{1}{Z_{\beta, \Lambda}^{\tau}} e^{-\beta H_{\Lambda}^{\tau}(\sigma)} \quad\left(\sigma \in \Omega_{\Lambda}\right),
$$

where $\beta$ is the inverse of the temperature (i.e. $\beta=1 / T$ ) and the partition function $Z_{\beta, \Lambda}^{\tau}$ is a normalizing constant. When the boundary conditions are uniformly equal to +1 
(resp. -1 ) we will denote the Gibbs measure by $\pi_{\Lambda}^{+}$(resp. $\pi_{\Lambda}^{-}$). Throughout the paper we will omit the superscript $\tau$ and the subscript $\Lambda$ from the notation of the Gibbs measure $\pi$ when these are made clear from the context.

The Gibbs measure enjoys a useful monotonicity property that will play a key role in our analysis. Consider the usual partial order on $\Omega_{\Lambda}$ whereby $\sigma \leq \eta$ if $\sigma_{x} \leq \eta_{x}$ for all $x \in \Lambda$. A function $f: \Omega_{\Lambda} \rightarrow \mathbb{R}$ is increasing (resp. decreasing) if $\sigma \leq \eta$ implies $f(\sigma) \leq$ $f(\eta)$ (resp. $f(\sigma) \geq f(\eta)$ ). An event is increasing (resp. decreasing) if its characteristic function is increasing (resp. decreasing). Given two probability measures $\mu, v$ on $\Omega_{\Lambda}$ we say that $\mu$ is stochastically dominated by $v$, denoted by $\mu \preceq v$, if $\mu(f) \leq v(f)$ for all increasing functions $f$ (here and in what follows, $\mu(f)$ stands for $\left.\int f d \mu\right)$. According to this notation the well-known FKG inequalities [17] state that:

- If $\tau \leq \tau^{\prime}$ then $\pi_{\Lambda}^{\tau} \preceq \pi_{\Lambda}^{\tau^{\prime}}$.

- If $f$ and $g$ are increasing then $\pi_{\Lambda}^{\tau}(f g) \geq \pi_{\Lambda}^{\tau}(f) \pi_{\Lambda}^{\tau}(g)$.

The phase transition regime in the 2D Ising model occurs at low temperature and it is characterized by spontaneous magnetization in the thermodynamic limit. There is a critical value $\beta_{c}$ such that for all $\beta>\beta_{c}$,

$$
\lim _{\Lambda \rightarrow \mathbb{Z}^{2}} \pi_{\Lambda}^{+}\left(\sigma_{0}\right)=-\lim _{\Lambda \rightarrow \mathbb{Z}^{2}} \pi_{\Lambda}^{-}\left(\sigma_{0}\right)=m_{\beta}>0
$$

Furthermore, in the thermodynamic limit the measures $\pi_{\Lambda}^{+}$and $\pi_{\Lambda}^{-}$converge (weakly) to two distinct Gibbs measures $\pi_{\infty}^{+}$and $\pi_{\infty}^{-}$which are measures on the space $\Omega_{\mathbb{Z}^{2}}$, each representing a pure state. We will focus on this phase-coexistence region $\beta>\beta_{c}$.

The Glauber dynamics for the Ising model is a family of continuous-time Markov chains on the state space $\Omega_{\Lambda}$, reversible with respect to the Gibbs distribution $\pi_{\Lambda}^{\tau}$. An important and natural example of this stochastic dynamics is the heat-bath dynamics, which we will now describe, postponing the formulation of the general Glauber dynamics to $\$ 2.1$. Note that our results apply to all of these chains (e.g., Metropolis etc.) by standard arguments for comparing their mixing times (see e.g. [31]).

The heat-bath dynamics for the Ising model $\Omega_{\Lambda}$ is defined as follows. With a rate one independent Poisson process for each vertex $x$, the spin $\sigma_{x}$ is refreshed by sampling a new value from the set $\{-1,+1\}$ according to the conditional Gibbs measure

$$
\pi_{\sigma, x}^{\tau}:=\pi_{\Lambda}^{\tau}\left(\cdot \mid \sigma_{y}, y \neq x\right) .
$$

It is easy to verify that the heat-bath chain is indeed reversible with respect to $\pi_{\Lambda}^{\tau}$ and is characterized by the generator

$$
\left(\mathcal{L}_{\Lambda}^{\tau} f\right)(\sigma)=\sum_{x \in \Lambda}\left[\pi_{\sigma, x}^{\tau}(f)-f(\sigma)\right]
$$

where $\pi_{\sigma, x}^{\tau}(f)$ is the average of $f$ with respect to the conditional Gibbs measure $\pi_{\sigma, x}^{\tau}$ acting only on the variable $\sigma_{x}$. The Dirichlet form associated to $\mathcal{L}_{\Lambda}^{\tau}$ takes the form

$$
\mathcal{E}_{\Lambda}^{\tau}(f, f)=\sum_{x \in \Lambda} \pi_{\Lambda}^{\tau}\left(\operatorname{Var}_{\sigma, x}^{\tau}(f)\right)
$$


where $\operatorname{Var}_{\sigma, x}^{\tau}(f)$ denotes the variance with respect to $\pi_{\sigma, x}^{\tau}$. It is possible to extend the above definition of the generator $\mathcal{L}_{\Lambda}^{\tau}$ directly to the whole lattice $\mathbb{Z}^{2}$ and get a well-defined Markov process on $\Omega_{\mathbb{Z}^{2}}$ (see e.g. [29]). The latter will be referred to as the infinite volume Glauber dynamics, with generator denoted by $\mathcal{L}_{\mathbb{Z}^{2}}$.

We will denote by $\mu_{t}^{\sigma}$ the distribution of the chain at time $t$ when the starting configuration is identically equal to $\sigma$. For instance, for any $f$ and $\sigma$ the expectation of $f$ with respect to $\mu_{t}^{\sigma}$ is given by $\left(T_{\Lambda}^{\tau}(t)\right) f(\sigma)$ where $T_{\Lambda}^{\tau}$ is the Markov semigroup generated by $\mathcal{L}_{\Lambda}^{\tau}$. The notation $\mu_{t}^{ \pm}$will stand for the corresponding quantity for an initial configuration of either all-plus or all-minus.

A key quantity that measures the rate of convergence of Glauber dynamics to stationarity is the gap in the spectrum of its generator, denoted by gap $\tau_{\Lambda}^{\tau}$. The Dirichlet form associated with $\mathcal{L}_{\Lambda}^{\tau}$ yields the following characterization for the spectral gap:

$$
\operatorname{gap}_{\Lambda}^{\tau}=\inf _{f} \frac{\mathcal{E}_{\Lambda}^{\tau}(f, f)}{\operatorname{Var}_{\Lambda}^{\tau}(f)},
$$

where the infimum is over all nonconstant $f \in L^{2}\left(\pi_{\Lambda}^{\tau}\right)$. Another useful measure for the speed of relaxation to equilibrium is the total-variation mixing time, which is defined as follows. Recall that the total-variation distance between two measures $\phi, \psi$ on a finite probability space $\Omega$ is defined as

$$
\|\phi-\psi\|:=\sup _{A \subset \Omega}|\phi(A)-\psi(A)|=\frac{1}{2} \sum_{x \in \Omega}|\phi(x)-\psi(x)| .
$$

For any $\varepsilon \in(0,1)$, the $\varepsilon$-mixing time of the Glauber dynamics is given by

$$
T_{\mathrm{MIX}}(\varepsilon):=\inf \left\{t>0: \sup _{\sigma \in \Omega_{\Lambda}}\left\|\mu_{t}^{\sigma}-\pi_{\Lambda}^{\tau}\right\| \leq \varepsilon\right\} .
$$

When $\varepsilon=1 /(2 e)$ we will simply write $T_{\mathrm{MIX}}$. This particular definition yields the following well-known inequalities (see e.g. [27,45]):

$$
\begin{aligned}
\sup _{\sigma \in \Omega_{\Lambda}}\left\|\mu_{t}^{\sigma}-\pi_{\Lambda}^{\tau}\right\| \leq \exp \left(-\left\lfloor t / T_{\mathrm{MIX}}\right\rfloor\right) & \text { for all } t \geq 0, \\
\frac{1}{\text { gap }} \leq T_{\mathrm{MIX}} \leq \frac{1}{\text { gap }} \log \frac{2 e}{\pi_{\min }} & \text { where } \pi_{\min }=\min _{\sigma \in \Omega_{\Lambda}} \pi_{\Lambda}^{\tau}(\sigma) .
\end{aligned}
$$

The last inequality shows that in our setting $\mathrm{gap}^{-1}$ and $T_{\mathrm{MIX}}$ are always within a factor of $O(|\Lambda|)$ from one another (to see this, observe that $\pi_{\Lambda}^{\tau}(\sigma) / \pi_{\Lambda}^{\tau}\left(\sigma^{\prime}\right) \leq \exp (O(|\Lambda|))$ for any $\sigma, \sigma^{\prime} \in \Omega_{\Lambda}$ by (1.1) whereas $\left.\left|\Omega_{\Lambda}\right|=2^{|\Lambda|}\right)$. One could restate our results as well as the analogous conjecture on the polynomial mixing time under all-plus b.c. in terms of $\mathrm{gap}^{-1}$ (expected to have order $L$, the side-length of $\Lambda$, for any $\beta>\beta_{c}$; see $[5,9]$ ).

\subsection{Main results}

We are now in a position to formalize the main contribution of this paper. The following theorem is the counterpart of the main result obtained by two of the present authors in [37]. Here we feature an improved estimate that in addition holds not only for large enough $\beta$ but throughout the phase-coexistence region. 
Theorem 1. For any $\beta>\beta_{c}$ there exists some $c(\beta)>0$ so that the following holds for the Glauber dynamics for the Ising model on the square $\Lambda_{L}$ at inverse temperature $\beta$. If $L$ is of the form $L=2^{n}-1$ for some integer $n$ then:

(1) If the boundary conditions $\tau$ are sampled from a law $\mathbf{P}$ that either stochastically dominates the pure phase $\pi_{\infty}^{+}$or is stochastically dominated by $\pi_{\infty}^{-}$then

$$
\mathbf{E}\left\|\mu_{t_{L}}^{ \pm}-\pi^{\tau}\right\| \leq c / L \quad \text { for } t_{L}=L^{c \log L} .
$$

In particular,

$$
\mathbf{P}\left(T_{\mathrm{MIX}} \geq L^{c \log L}\right) \leq c / L .
$$

(2) The estimates (1.3), (1.4) also hold if $\mathbf{P}$ is stochastically dominated by $\pi_{\infty}^{-}$on one side of $\Lambda_{L}$ and stochastically dominates $\pi_{\infty}^{+}$on the union of the other three sides. A similar statement holds if the roles of $(+)$ and $(-)$ are reversed.

The most natural consequence of the above result is obtained when $\mathbf{P}$ concentrates on homogeneous boundary conditions, where the best previous bounds were $\exp \left(O\left(L^{\varepsilon}\right)\right)$ for any $\varepsilon>0$ and $\beta$ large enough ([37]) along with $\exp (o(L))$ for all other $\beta>\beta_{c}$ ([11]).

Corollary 2. For any $\beta>\beta_{c}$ there exists some $c(\beta)>0$ so that the mixing time of Glauber dynamics for the Ising model on the square $\Lambda_{L}$ with b.c. $\tau \equiv+1$ satisfies

$$
T_{\mathrm{MIX}} \leq L^{c \log L} \text {. }
$$

The same bound holds if the boundary conditions are $(+)$ on three sides and $(-)$ on the remaining one, and similarly if $(+)$ is replaced by $(-)$.

We believe that improving the above $L^{c \log L}$ bound into the conjectured polynomial would require substantially new ideas; cf. also the discussion in $\$ 4.2$. Indeed, in the present recursive framework in which the final scale of the system is reached via a doubling sequence, at each step the mixing-time estimate worsens by a power of $L$ (hence the quasi-polynomial bound). For a polynomial upper bound one could not afford to lose more than a constant factor on average along these steps.

One may also apply Theorem 1 to deduce the mixing behavior of the 2D Ising model under Bernoulli boundary conditions, as illustrated by the next corollary. Here and in what follows we say that an event holds with high probability (w.h.p.) whenever its probability tends to 1 as the size of the system tends to $\infty$.

Corollary 3. Let $\beta>\beta_{c}$ and consider Glauber dynamics for the Ising model on the square $\Lambda_{L}$ with b.c. $\tau$ consisting of i.i.d. Bernoulli variables, $\mathbf{P}(\tau(x)=+1)=p_{+}$for some $p_{+} \geq \frac{1}{2}(1+\tanh (4 \beta))$. Then w.h.p. $T_{\mathrm{MIX}} \leq L^{c \log L}$ for some $c(\beta)>0$.

To obtain the above corollary observe that the Bernoulli boundary conditions with the above specified $p^{+}$clearly stochastically dominate the marginal of $\pi_{\infty}^{+}$on $\partial \Lambda$.

The mixing time of Glauber dynamics for Ising on a finite box under all-plus b.c. is closely related to the asymptotic decay of the time autocorrelation function in the infinitevolume dynamics on $\mathbb{Z}^{2}$ started at the plus phase. Here it was conjectured in [15] that the 
decay should follow a stretched exponential of the form $\exp (-c \sqrt{t})$. As a by-product of Corollary 2 (and standard monotonicity arguments) we obtain a new bound on this quantity, improving on the previous estimate due to [37] of $\exp \left(-(\log t)^{c}\right)$ with arbitrarily large $c$ which was applicable for large enough $\beta$.

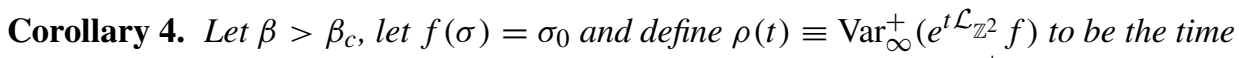
autocorrelation of the spin at the origin started from the plus phase $\pi_{\infty}^{+}$(the variance is with respect to the plus phase $\left.\pi_{\infty}^{+}\right)$. Then there exists some $c(\beta)>0$ such that for any $t$,

$$
\rho(t) \leq \exp \left(-e^{c \sqrt{\log t}}\right)
$$

\subsection{Related work}

Over the last two decades considerable effort was devoted to the formidable problem of establishing polynomial mixing for the stochastic Ising model on a finite lattice with all-plus b.c. Following is a partial account of related results.

Analogous to its conjectured behavior on $\mathbb{Z}^{2}$, the mixing of Glauber dynamics for the Ising model on the lattice $\mathbb{Z}^{d}$ in any fixed dimension $d$ is believed to be polynomial in the side-length of the box at any temperature in the presence of an all-plus boundary. Unfortunately, the state-of-the-art rigorous analysis of the problem in three dimensions and higher is far more limited. Faced with the polynomial lower bounds of [5], the best known upper bound for dimension $d \geq 3$ is $\exp \left(L^{d-2+o(1)}\right)$ for large enough $\beta$ (as usual $L$ being the side-length) due to [48]. Compare this with the case of no (i.e. free) boundary conditions case where it was shown in [49] that $\mathrm{gap}^{-1}$ (and thus also $T_{\text {MIX }}$ ) is at least $c \exp \left(c^{\prime} \beta L^{d-1}\right)$ for some $c=c(\beta)>0$ and an absolute constant $c^{\prime}>0$.

In two dimensions, ever since the work of Martinelli [32] in 1994 (an upper bound of $\exp \left(L^{1 / 2+o(1)}\right)$ at low enough temperatures) and until quite recently no real progress has been made on the original problem. Nevertheless, various variants of this problem have become fairly well understood. For instance, nearly homogeneous boundary conditions were studied in [2,3]. Analogues of the problem on non-amenable geometries (in terms of a suitable parameter measuring the growth of balls to replace the side-length) were established, pioneered by the work of [36] on trees and followed by results of [4] on a class of hyperbolic graphs of large degrees. The one-dimensional Solid-On-Solid model (SOS), proposed as an idealization of the behavior of Ising contours at low temperatures, was studied in [35] where the authors obtained several insights into the evolution of the contours.

Finally, the conjectured Lifshitz behavior of $O\left(L^{2}\right)$ was confirmed at zero temperature, in both dimensions two $[9,12,16]$ and three [9] and also for the one-dimensional SOS model [10], with the recent work [9] providing sharp bounds also for near-zero temperatures (namely when $\beta \geq c \log L$ for a suitably large $c>0$ ). This set of works represents a first step towards the rigorous justification of the above mentioned mean-curvature heuristics.

As mentioned above, the $\exp \left(L^{1 / 2}\right)$ barrier was finally broken in the recent paper [37], replacing it by $\exp \left(c L^{\varepsilon}\right)$ for an arbitrarily small $\varepsilon>0$ and sufficiently large $\beta$ (where the 
constant $c=c(\beta, \varepsilon)$ diverges to $+\infty$ as $\varepsilon \rightarrow 0)$. At the heart of the proof of the main result of that paper ([37, Theorem 1.6]) was an inductive procedure which will serve as our main benchmark here. We will shortly review that argument in $\S 3$ in order to motivate and better understand the new steps gained in the present work.

Finally, there is an extensive literature on the phase-separation lines in the 2D Ising model, going back to $[1,18]$. In $\S 2$ we will review the tools we will need from the randomline representation framework of [43,44]. For further information see e.g. [42] and the references therein, and also the more recent developments [7,8] where duality techniques are used to put the Ornstein-Zernike theory for the Ising two-point function on rigorous grounds.

\section{Preliminaries}

\subsection{General Glauber dynamics}

The class of Glauber dynamics for the Ising model on a finite box $\Lambda \subset \mathbb{Z}^{2}$ consists of the continuous-time Markov chains on the state space $\Omega_{\Lambda}$ that are given by the generator

$$
\left(\mathcal{L}_{\Lambda}^{\tau} f\right)(\sigma)=\sum_{x \in \Lambda} c(x, \sigma)\left(f\left(\sigma^{x}\right)-f(\sigma)\right),
$$

where $\sigma^{x}$ is the configuration $\sigma$ with the spin at $x$ flipped and the transition rates $c(x, \sigma)$ should satisfy the following conditions:

(1) Finite range interactions: For some fixed $R>0$ and any $x \in \Lambda$, if $\sigma, \sigma^{\prime} \in \Omega_{\Lambda}$ agree on the ball of diameter $R$ about $x$ then $c(x, \sigma)=c\left(x, \sigma^{\prime}\right)$.

(2) Detailed balance: For all $\sigma \in \Omega_{\Lambda}$ and $x \in \Lambda$,

$$
\frac{c(x, \sigma)}{c\left(x, \sigma^{x}\right)}=\exp \left(-\beta \nabla_{x} H_{\Lambda}^{\tau}(\sigma)\right)
$$

where $\nabla_{x} H_{\Lambda}^{\tau}(\sigma)=H_{\Lambda}^{\tau}\left(\sigma^{x}\right)-H_{\Lambda}^{\tau}(\sigma)=2 \sigma_{x}\left[\sum_{y \in \Lambda, y \sim x} \sigma_{y}+\sum_{y \in \partial \Lambda, y \sim x} \tau_{y}\right]$.

(3) Positivity and boundedness: The rates $c(x, \sigma)$ are uniformly bounded from below and above by some fixed $C_{1}, C_{2}>0$.

(4) Translation invariance: If $\sigma \equiv \sigma^{\prime}(\cdot+\ell)$, where $\ell \in \Lambda$ and addition is according to the lattice metric, then $c(x, \sigma)=c\left(x+\ell, \sigma^{\prime}\right)$ for all $x \in \Lambda$.

The Glauber dynamics generator with such rates defines a unique Markov process, reversible with respect to the Gibbs measure $\mu_{\Lambda}^{\tau}$. The two most notable examples for the choice of transition rates are

(i) Metropolis: $c(x, \sigma)=\exp \left(-\beta \nabla_{x} H_{\Lambda}^{\tau}(\sigma)\right) \wedge 1$.

(ii) Heat-bath: $c(x, \sigma)=\left[1+\exp \left(\beta \nabla_{x} H_{\Lambda}^{\tau}(\sigma)\right)\right]^{-1}$.

See e.g. [31] for standard comparisons between these chains, in particular implying that their individual mixing times are within a factor of at most $O(|\Lambda|)$ from one another (hence our results apply to every one of these chains). 


\subsection{Surface tension}

Denote by $\tau_{\beta}(\theta)$ the surface tension that corresponds to the angle $\theta$, defined as follows. Associate with each angle $\theta \in[-\pi / 4, \pi / 4]$ the unit vector $\vec{n}_{\theta}=(\cos \theta, \sin \theta) \in \mathbb{S}^{1}$ and the following b.c. $\eta_{\theta}$ for $\Lambda_{L}=\{-\lfloor L / 2\rfloor, \ldots,\lfloor L / 2\rfloor\}^{2}$ :

$$
\eta_{\theta}(v)= \begin{cases}+1 & \text { if }\left\langle v, \vec{n}_{\theta}\right\rangle>0, \\ -1 & \text { if }\left\langle v, \vec{n}_{\theta}\right\rangle>\leq 0 .\end{cases}
$$

Let $Z_{\beta, \Lambda_{L}}^{\eta_{\theta}}$ be the partition-function of the corresponding Ising model and, as usual, let $Z_{\beta, \Lambda_{L}}^{+}$denote the partition-function under the all-plus b.c. The surface tension in the direction orthogonal to $\vec{n}_{\theta}$ is the limit

$$
\tau_{\beta}(\theta)=-\lim _{L \rightarrow \infty} \frac{\cos \theta}{L} \log \frac{Z_{\beta, \Lambda_{L}}^{\eta_{\theta}}}{Z_{\beta, \Lambda_{L}}^{+}},
$$

which gives rise to an even analytic function $\tau_{\beta}$ with period $\pi / 2$ on $\mathbb{R}$ (a closed formula appears e.g. in [44, Section 5]). One can then extend the definition of $\tau_{\beta}$ to $\mathbb{R}^{2}$ by homogeneity, setting $\tau_{\beta}(x)=\tau_{\beta}\left(\theta_{x}\right)|x|$, where $|x|$ denotes the Euclidean norm of $x$ and $\theta_{x}$ is the angle it forms with $(1,0)$. For all $\beta>\beta_{c}$ this qualifies as a norm on $\mathbb{R}^{2}$.

The surface tension measures the effect of the interface induced by the boundary conditions $\eta_{\theta}$ on the free energy and thus plays an important role in the geometry of the low temperature Ising model. For instance, it was shown in [46] that the large deviations of the magnetization in a square are governed by $\tau_{\beta}(0)$ (also see [24, 25]).

One of the useful properties of the surface tension is the sharp triangle inequality (see for instance [44, Proposition 2.1]): For any $\beta>\beta_{c}$ there exists a strictly positive constant $\kappa_{\beta}$ such that for any $x, y \in \mathbb{R}^{2}$ we have

$$
\tau_{\beta}(x)+\tau_{\beta}(y)-\tau_{\beta}(x+y) \geq \kappa_{\beta}(|x|+|y|-|x+y|),
$$

A thorough account of additional properties of the surface tension may be found e.g. in [14] and [42].

\subsection{Duality}

Let $\mathbb{Z}^{2 *}:=\mathbb{Z}^{2}+(1 / 2,1 / 2)$ denote the dual lattice to $\mathbb{Z}^{2}$. The collection of edges of $\mathbb{Z}^{2}$ and of $\mathbb{Z}^{2 *}$ will be denoted by $E_{\mathbb{Z}^{2}}$ and $E_{\mathbb{Z}^{2 *}}^{*}$ respectively. It is useful to identify an edge $e=(x, y) \in E_{\mathbb{Z}^{2}}$ with the closed unit segment in $\mathbb{R}^{2}$ whose endpoints are $\{x, y\}$, and similarly for edges in $E_{\mathbb{Z}^{2 *}}^{*}$. To each edge $e=(x, y) \in E_{\mathbb{Z}^{2}}$ there corresponds a unique dual edge $e^{*} \in E_{\mathbb{Z}^{2 *}}^{*}$ defined by the condition $e \cap e^{*} \neq \emptyset$.

Given a finite box $\Lambda \subset \mathbb{Z}^{2}$ of the form $\Lambda=\left\{x=\left(x_{1}, x_{2}\right) \in \mathbb{Z}^{2}:\left|x_{1}\right| \leq L_{1}\right.$, $\left.\left|x_{2}\right| \leq L_{2}\right\}$, the dual box $\Lambda^{*} \subset \mathbb{Z}^{2 *}$ is $\Lambda^{*}=\left\{x=\left(x_{1}, x_{2}\right) \in \mathbb{Z}^{2 *}:\left|x_{1}\right| \leq L_{1}+1 / 2\right.$, $\left.\left|x_{2}\right| \leq L_{2}+1 / 2\right\}$. The set of dual edges of $\Lambda^{*}$, denoted by $E_{\Lambda^{*}}^{*}$, is the set of dual edges for which both endpoints lie in $\Lambda^{*}$. Notice that for each edge $e=(x, y) \in E_{\mathbb{Z}^{2}}$ such that $\{x, y\} \cap \Lambda \neq \emptyset$, the corresponding dual edge $e^{*}$ necessarily belongs to $E_{\Lambda^{*}}^{*}$. These definitions readily generalize to an arbitrary finite $\mathcal{G} \subset \mathbb{Z}^{2}$, in which case $\mathcal{G}^{*} \subset \mathbb{Z}^{2 *}$ consists of all dual sites whose $L^{1}$-distance from $\mathcal{G}$ equals 1 . 
For any $\beta>\beta_{c}$ we associate the dual inverse temperature $\beta^{*}$ via the duality relation $\tanh \left(\beta^{*}\right)=e^{-2 \beta}$. Notice that for any $\beta>\beta_{c}$ the dual inverse temperature $\beta^{*}$ lies below $\beta_{c}$ which is the unique fixed point of the map $\beta \mapsto \beta^{*}$. We will often refer to the Gibbs measure on a subset of the dual lattice $\Lambda^{*}$ at the inverse temperature $\beta^{*}$ under free boundary, denoting it by $\pi_{\Lambda^{*}}^{*}$. The following well-known fact addresses the exponential decay of the two-point correlation function for the free Ising Gibbs measure above the critical temperature.

Lemma 2.1 (e.g. [38, p. 309, Eq. (4.39)], together with the GKS inequalities [21, 26]). Let $\Lambda \subset \mathbb{Z}^{2}$ and $\beta>\beta_{c}$. There exists some $C_{\beta}>0$ such that for any $x, y \in \Lambda^{*}$,

$$
\pi_{\Lambda^{*}}^{*}\left(\sigma_{x} \sigma_{y}\right) \leq\left(\frac{C_{\beta}}{\sqrt{x-y}} \wedge 1\right) \exp \left(-\tau_{\beta}(x-y)\right)
$$

A matching exponent for the spin-spin correlation was established by [20] for two opposite points in the (dual) infinite strip. Let $S=\{1, \ldots, \ell\} \times \mathbb{Z}$ for some integer $\ell$ and fix $\beta>\beta_{c}$. In the dual $S^{*}$ we let $x=(1 / 2,1 / 2)$ and $y=(\ell+1 / 2,1 / 2)$ and consider the free Gibbs measure at inverse temperature $\beta^{*}$. It was shown in [20, formula (2.22)] that in this setting there exists some $c_{\beta}>0$ such that

$$
\pi_{S^{*}}^{*}\left(\sigma_{x} \sigma_{y}\right)=\frac{c_{\beta}+o(1)}{\sqrt{\ell}} \exp \left(-\tau_{\beta}(0) \ell\right)
$$

where the $o(1)$-term tends to 0 as $\ell \rightarrow \infty$.

\subsection{Contours}

Let $\mathcal{G}=(V, E)$ be a finite subgraph of $\left(\mathbb{Z}^{2 *}, E_{\mathbb{Z}^{2 *}}^{*}\right)$. The boundary of a subset $B \subset E$ of dual edges, denoted by $\delta B$, is the set of vertices of $V$ with an odd number of adjacent edges of $B$. If $\delta B=\emptyset$ we say that $B$ is closed, otherwise it is open.

A chain of sites of length $k$ from $x$ to $y$ in $\mathcal{G}$ has the standard definition of a sequence of sites $x=u_{0}, u_{1}, \ldots, u_{k}=y$ such that $u_{i} \in \mathcal{G}$ and $\left|u_{i}-u_{i-1}\right|=1$ for all $i$. A $*$-chain from $x$ to $y$ is similarly defined with the exception that the distance requirement is relaxed to $1 \leq\left|u_{i}-u_{i-1}\right| \leq \sqrt{2}$ for all $i$. A path from $x$ to $y$ in $B$ is a chain of sites consisting of edges of $B$, that is, $\left(u_{i-1}, u_{i}\right) \in B$ for all $i$. We say that a path is closed if its endpoint and starting point coincide, otherwise we say that it is open.

A set $B \subset E$ of dual edges can be uniquely partitioned into a finite number of edgedisjoint simple lines in $E \cap \mathbb{R}^{2}$ called contours. This is achieved by repeating the following procedure referred to as the South-East (SE) splitting rule: When four bonds meet at a vertex we separate them along the SE-oriented diagonal going through the intersection. Alternatively, one may globally apply the $S W$ splitting rule, analogously defined with the South-West orientation replacing the South-East one (see Figure 1).

Contours can be either open or closed (with the same distinction as in paths). The length of a contour $\gamma$, denoted by $|\gamma|$, is the number of edges in $\gamma$, and the length of a collection $\gamma=\left\{\gamma_{1}, \ldots, \gamma_{k}\right\}$ of contours will simply be the sum of all the individual lengths. Given a finite family $\underline{\gamma}=\left\{\gamma_{1}, \ldots, \gamma_{k}\right\}$ of contours we say that it is compatible if it is the contour decomposition of its collection of dual edges $\bigcup_{i} \gamma_{i}$. We further say that 


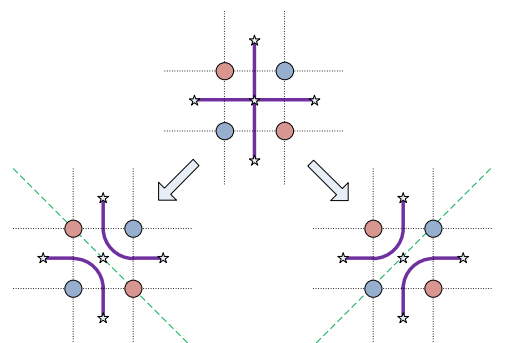

Fig. 1. SE and SW splitting-rules for forming the contours.

$\gamma$ is $E$-compatible (or $\mathcal{G}$-compatible) to emphasize that in addition all the edges of $\bigcup_{i} \gamma_{i}$ belong to $E$, the edge-set of $\mathcal{G}$.

Given boundary conditions $\tau \in\{-1,1\}^{\mathbb{Z}^{2}}$ and a box $\Lambda$, each spin-configuration $\sigma$ compatible with $\tau$ outside $\Lambda$ (i.e. $\sigma_{x}=\tau_{x}$ for any $x \notin \Lambda$ ) can be uniquely specified by giving all the edges $e=(x, y) \in E_{\mathbb{Z}^{2}}$ such that $\sigma_{x} \neq \sigma_{y}$ and $\{x, y\} \cap \Lambda \neq \emptyset$ (that is, all edges whose endpoint sites disagree). Equivalently, one can specify the corresponding dual edges of $\Lambda^{*}$. By applying the above contour decomposition we see that each configuration $\sigma$ compatible with $\tau$ is uniquely characterized by its collection of closed and open contours (see Figure 2 for an illustration). The open contours obtained in this manner are called the phase-separation lines.

It is clear that the boundary $\delta \underline{\lambda}$ of the open contours belongs to $\partial \Lambda^{*}$ and must coincide with a certain set $V(\tau)$ uniquely specified by the boundary conditions $\tau$ (i.e. independent
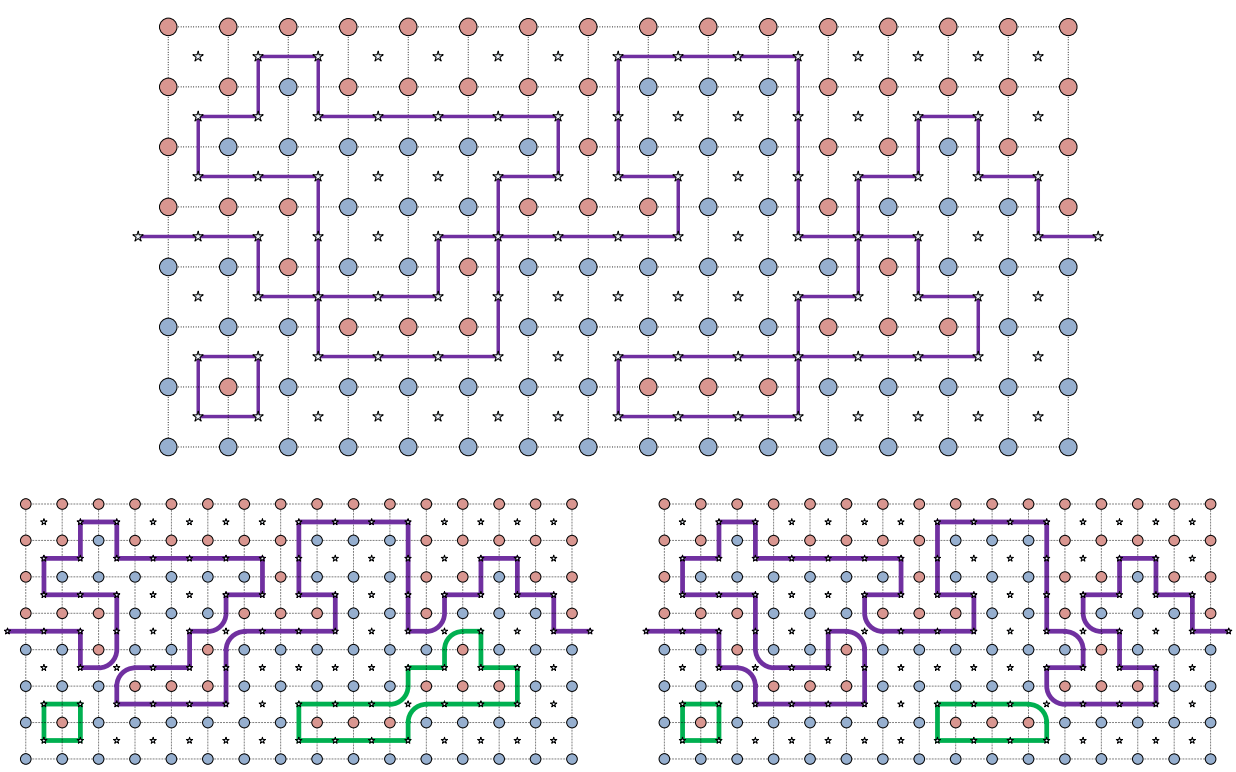

Fig. 2. Contour decomposition of the edge-set induced by an Ising configuration on a box with mixed boundary conditions according to both SE and SW splitting rules. 
of the values $\sigma$ gives to the spins of $\Lambda$ ). Notice that the cardinality of $V(\tau)$, if different from zero, must be even.

A family $\underline{\gamma}$ of closed and open simple lines is called $\tau$-compatible if there exists a configuration $\bar{\sigma}$ compatible with $\tau$ in $\Lambda$ from which $\underline{\gamma}$ is obtained in the above procedure. One can easily verify that when $\Lambda$ is a box, the set of $\tau$-compatible contours coincides with the set of $E_{\Lambda^{*}}^{*}$-compatible contours whose boundary is equal to $V(\tau)$.

\subsection{Random-line representation}

For a finite subgraph $\mathcal{G}=(V, E)$ of $\left(\mathbb{Z}^{2 *}, E_{\mathbb{Z}^{2 *}}^{*}\right)$ and an $E$-compatible family $\underline{\theta}$ of contours, two different partition functions $Z(\mathcal{G})$ and $Z(\mathcal{G} \mid \underline{\theta})$ will turn out to be useful for a given $\beta>0$ :

$$
\begin{aligned}
& Z(\mathcal{G})=\sum_{\substack{\gamma \\
\text { is } \underline{\underline{\gamma}} \bar{E} \text {-compatible }}} e^{-2 \beta|\underline{\gamma}|}, \\
& Z(\mathcal{G} \mid \underline{\theta})=\sum_{\substack{\underline{\gamma} \cup \underline{\theta}: \delta \text { is } E \text {-compatible }}} e^{-2 \beta|\underline{\gamma}|} .
\end{aligned}
$$

Using $Z(\mathcal{G})$ and $Z(\mathcal{G} \mid \underline{\theta})$ we define the weight (not necessarily a probability distribution) corresponding to the family $\underline{\theta}$ of contours, denoted by $q_{\mathcal{G}}(\underline{\theta})$, to be

$$
q_{\mathcal{G}}(\underline{\theta})= \begin{cases}\frac{Z(\mathcal{G} \mid \underline{\theta})}{Z(\mathcal{G})} e^{-2 \beta|\underline{\theta}|} & \text { if } \underline{\theta} \text { is } E \text {-compatible } \\ 0 & \text { otherwise. }\end{cases}
$$

The key reason for the above formula is the following random-line representation for even-point correlation functions: Consider the Ising model on $\mathcal{G}$ at inverse temperature $\beta^{*}$ and free boundary conditions. Let $\pi_{\mathcal{G}}^{*}$ be the associated Gibbs measure and let $A \subset V$ have even cardinality. Then the following holds (see [44, Lemma 6.9]):

$$
\sum_{\underline{\lambda}: \delta \underline{\lambda}=A} q_{\mathcal{G}}(\underline{\lambda})=\pi_{\mathcal{G}}^{*}\left(\prod_{x \in A} \sigma_{x}\right) .
$$

Remark. If the cardinality of $A$ is odd then the r.h.s. of (2.7) is zero by symmetry and the 1.h.s. is zero due to the definition of $q_{\mathcal{G}}(\underline{\lambda})$.

Back to the low temperature Ising model in a box $\Lambda$ with boundary condition $\tau$, let $\underline{\lambda}$ be a collection of $\tau$-compatible open contours. Then, by construction,

$$
\pi_{\Lambda}^{\tau}(\sigma: \underline{\lambda}(\sigma)=\underline{\lambda})=\frac{q_{\Lambda^{*}}(\underline{\lambda})}{\sum_{\underline{\lambda}^{\prime}: \delta \underline{\lambda}^{\prime}=V(\tau)} q_{\Lambda^{*}}\left(\underline{\lambda}^{\prime}\right)}=\frac{q_{\Lambda^{*}}(\underline{\lambda})}{\pi_{\Lambda^{*}}^{*}\left(\prod_{x \in V(\tau)} \sigma_{x}\right)},
$$

where with a slight abuse of notation we have identified $\Lambda^{*}$ with the graph $\mathcal{G}=\left(\Lambda^{*}, E_{\Lambda^{*}}^{*}\right)$ and in the last equality we used (2.7). The above formula will be the starting point of the proof of the new equilibrium estimates, Propositions 4.4 and 4.5 . 
We conclude this section with some of the main properties of the weights $q_{\mathcal{G}}(\underline{\lambda})$. For further information see [43,44].

Lemma 2.2 ([44, Lemma 6.3]). Let $\mathcal{G}=(V, E)$ be a finite subgraph of $\left(\mathbb{Z}^{2 *}, E_{\mathbb{Z}^{2 *}}^{*}\right)$ and let $\underline{\theta}$ be a family of $E$-compatible contours (open and closed). If $\mathcal{G}^{\prime}$ is a subgraph of $\mathcal{G}$ then $q_{\mathcal{G}^{\prime}}(\underline{\theta}) \geq q_{\mathcal{G}}(\underline{\theta})$.

Remark. Lemma 2.2 enables one to extend the definition of the weights $q_{\mathcal{G}}(\lambda)$ for finite contours $\lambda$ in an infinite graph $\mathcal{G}$ by taking the limit as $n \rightarrow \infty$ of $q_{\mathcal{G}_{n}}(\lambda)$, where $\mathcal{G}_{n}$ is the intersection of $\mathcal{G}$ with a box of size $n$. By Lemma 2.2 the sequence $q_{\mathcal{G}_{n}}(\lambda)$ is nonincreasing and non-negative hence its limit indeed exists.

Let $\mathcal{G}=(V, E)$ be a subgraph of $\left(\mathbb{Z}^{2 *}, E_{\mathbb{Z}^{2 *}}^{*}\right)$. The edge-boundary of an edge $e \in E$, denoted by $\Delta(e)$, consists of the edge $e$ itself together with any edge $e^{\prime} \in E$ that is incident to it and would belong to the same contour in the contour decomposition of $E$ via the agreed splitting rule. For instance, with the SE splitting rule the horizontal edge $e=[(x, y),(x+1, y)]$ in the dual lattice $\mathbb{Z}^{2 *}$ would have an edge-boundary of $\Delta(e)=$ $\{e,[(x, y),(x, y+1)],[(x+1, y),(x+1, y-1)]\}$. Given a subset $B \subset E$ of edges we define its edge-boundary as $\Delta(B)=\bigcup_{e \in B} \Delta(e)$. This definition implies that two contours $\lambda$ and $\gamma$, where $\lambda$ is closed and $\gamma$ is either open or closed, are $\mathcal{G}$-compatible if and only if the edge-set of $\lambda$ does not intersect $\Delta(\gamma)$ (see the related [44, Lemma 6.1]). The following lemma is a special case of [44, Lemma 6.4]):

Lemma 2.3 ([44, Eq. (6.17)]). Let $\mathcal{G}=(V, E)$ be a subgraph of $\left(\mathbb{Z}^{2 *}, E_{\mathbb{Z}^{2 *}}^{*}\right)$ and let $\underline{\theta}$ and $\underline{\lambda}$ denote two $\mathcal{G}$-compatible families of contours with corresponding edge-sets $E_{\underline{\theta}}$ and $E_{\underline{\lambda}}$ respectively. If $\underline{\lambda} \cup \underline{\theta}$ is $\mathcal{G}$-compatible (or equivalently if $\Delta(\underline{\lambda}) \cap E_{\underline{\theta}}=\emptyset$ ) then

$$
q_{\mathcal{G}}(\underline{\theta} \cup \underline{\lambda})=q_{\mathcal{G}_{\underline{\lambda}}}(\underline{\theta}) q_{\mathcal{G}}(\underline{\lambda}),
$$

where $\mathcal{G}_{\underline{\lambda}}$ is the subgraph of $\mathcal{G}$ given by the edge-set $E \backslash \Delta(\underline{\lambda})$.

We will frequently need estimates on the weight of a contour constrained to go through certain dual sites; to this end, the following definition will be useful. Let $\mathcal{G}=(V, E)$ and let $\lambda_{1}, \lambda_{2}$ be two open contours such that $\delta \lambda_{1}=\{x, y\}$ and $\delta \lambda_{2}=\{u, v\}$. We say that $\lambda_{1}, \lambda_{2}$ are disjoint if either they are $\mathcal{G}$-compatible, or their edge-sets are disjoint and the contour decomposition of the union of their edges is a single contour $\lambda$. Observe that in the latter case necessarily $\{x, y\} \cap\{u, v\} \neq \emptyset$. For a pair of disjoint open contours $\lambda_{1}, \lambda_{2}$ we write $\lambda_{1} \sqcup \lambda_{2}$ to denote either the collection $\left(\lambda_{1}, \lambda_{2}\right)$ in the former case, or the single contour $\lambda$ in the latter.

Lemma 2.4 ([44, Lemma 6.5]). Let $\mathcal{G}=(V, E)$ be a graph in the dual lattice $\mathbb{Z}^{2 *}$. For any $x, y, u, v \in V$,

$$
\sum_{\substack{\lambda=\lambda_{1} \sqcup \lambda_{2} \\ \delta \lambda_{1}=\{\bar{x}, y\}, \delta \lambda_{2}=\{u, v\}}} q_{\mathcal{G}}(\underline{\lambda}) \leq \sum_{\substack{\lambda_{1} \\ \delta \lambda_{1}=\{x, y\}}} q_{\mathcal{G}}\left(\lambda_{1}\right) \sum_{\substack{\lambda_{2} \\ \delta \lambda_{2}=\{u, v\}}} q_{\mathcal{G}}\left(\lambda_{2}\right) .
$$

In particular, we have 
Corollary 2.5 ([43, Eq. (5.29)]). Let $\mathcal{G}=(V, E)$ be a graph in the dual lattice $\mathbb{Z}^{2 *}$. For any $\beta>\beta_{c}$ and any $u, v, z \in V$,

$$
\sum_{\substack{\lambda: \delta \lambda=\{u, v\} \\ z \in \lambda}} q_{\mathcal{G}}(\lambda) \leq\left(\sum_{\lambda: \delta \lambda=\{u, z\}} q_{\mathcal{G}}(\lambda)\right)\left(\sum_{\lambda: \delta \lambda=\{z, v\}} q_{\mathcal{G}}(\lambda)\right)=\pi_{\mathcal{G}}^{*}\left(\sigma_{u} \sigma_{z}\right) \pi_{\mathcal{G}}^{*}\left(\sigma_{v} \sigma_{z}\right) .
$$

Together with Lemma 2.1 the above lemma immediately implies an upper bound on the weights in mention in terms of the surface tensions $\tau_{\beta}(u-v)$ and $\tau_{\beta}(v-z)$. The next lemma provides an analogous bound for the weights of closed contours going through a set of prescribed sites.

Lemma 2.6 ([43, Lemma 5.5(ii)]). Let $\mathcal{G}=(V, E)$ be a graph in $\mathbb{Z}^{2 *}$. Let $x_{1}, \ldots, x_{k}$ $\in V$ and identify $x_{0} \equiv x_{k}$. Then

$$
\sum_{\substack{\lambda: \delta \lambda=\emptyset \\ x_{1}, \ldots, x_{k} \in \lambda}} q_{\mathcal{G}}(\lambda) \leq \exp \left(-\sum_{i=1}^{k} \tau_{\beta}\left(x_{i}-x_{i-1}\right)\right) .
$$

\section{Inductive framework for rectangles with "plus" boundaries}

In this section we outline the recursive scheme developed in [37] which, as mentioned in $\S 1$, established a significantly improved upper bound of $\exp \left(c L^{\varepsilon}\right)$ for the mixing time on a box of side-length $L$ with "plus" b.c. at sufficiently low temperatures.

Given $\varepsilon>0$ (to be thought of as very small) and $L \in \mathbb{N}$ let

$$
R_{L}=\left\{x=(i, j) \in \mathbb{Z}^{2}: 1 \leq i \leq L, 1 \leq j \leq\left\lceil L^{1 / 2+\varepsilon}\right\rceil\right\} .
$$

Similarly one defines the rectangle $Q_{L}$, the only difference being that the vertical sides contain now $\left\lceil(2 L+1)^{1 / 2+\varepsilon}\right\rceil$ sites.

Definition 3.1. A distribution $\mathbf{P}$ of b.c. for a rectangle $R$ (which will be $R_{L}, Q_{L}$ or some translation of them) is said to belong to $\mathcal{D}(R)$ if its marginal on the union of North, East and West borders of $R$ is stochastically dominated by (the marginal of) the minus phase $\pi_{\infty}^{-}$of the infinite system, while the marginal on the South border of $R$ dominates the (marginal of the) infinite plus phase $\pi_{\infty}^{+}$.

The most natural example is to take $\mathbf{P}$ concentrated on the boundary condition $\tau \equiv-1$ on the North, East and West borders, and $\tau \equiv+1$ on the South border.

Definition 3.2. For any given $L \in \mathbb{N}, \delta>0, t>0$ consider the Ising model in $R_{L}$, with boundary condition $\tau$ chosen from some distribution $\mathbf{P}$. We say that $\mathcal{A}(L, t, \delta)$ holds if

$$
\mathbf{E}\left\|\mu_{t}^{ \pm}-\pi^{\tau}\right\| \leq \delta
$$

for every $\mathbf{P} \in \mathcal{D}\left(R_{L}\right)$. The statement $\mathcal{B}(L, t, \delta)$ is defined similarly, the only difference being that the rectangle $R_{L}$ is replaced by $Q_{L}$ (and $\mathbf{P}$ is required to belong to $\mathcal{D}\left(Q_{L}\right)$ ). 
With these definitions the iterative scheme developed in [37] can be summarized as follows.

Proposition 3.3 (The starting point). For every $\beta$ (thus not necessarily large) there exists $c=c(\beta)$ such that for every $L \in \mathbb{N}$ the statements $\mathcal{A}\left(L, t, e^{-t e^{-c L^{1 / 2+\varepsilon}}}\right)$ and $\mathcal{B}\left(L, t, e^{-t e^{-c L^{1 / 2+\varepsilon}}}\right)$ hold.

Remark. Notice that the factor $e^{-c L^{1 / 2+\varepsilon}}$ in front of the time $t$ is nothing but the negative exponential of the shortest side of the rectangle.

Theorem 3.4 (The inductive step). For every $\beta$ large enough there exist constants $c_{1}, c_{2}$, $c_{3}$ such that

$$
\mathcal{A}(L, t, \delta) \Rightarrow \mathcal{B}\left(L, t_{1}, \delta_{1}\right) \Rightarrow \mathcal{A}\left(2 L+1, t_{2}, \delta_{2}\right)
$$

where

$$
\begin{array}{ll}
\delta_{1}=c_{1}\left(\delta+e^{-c_{2} L^{2 \varepsilon}}+L^{2} e^{-c_{2} \log t}\right), & t_{1}=2 t, \\
\delta_{2}=c_{1}\left(\delta_{1}+e^{-c_{2} L^{3 \varepsilon}}\right)=c_{3}\left(\delta+e^{-c_{2} L^{2 \varepsilon}}+L^{2} e^{-c_{2} \log t}\right), & t_{2}=e^{c_{3} L^{3 \varepsilon}} t_{1}=2 e^{c_{3} L^{3 \varepsilon}} t .
\end{array}
$$

Remark. In the original statement in [37] the obvious requirement of $\beta$ large was missing due to a typo.

Corollary 3.5 (Solving the recursion). In the same setting of Theorem 3.4, for every $L \in\left\{2^{n}-1\right\}_{n \in \mathbb{N}}$ there exists

$$
\Delta(L) \leq \exp \left(-c^{\prime} L^{\varepsilon^{2}}\right)
$$

such that $\mathcal{A}(L, t, \Delta(L))$ holds for every $t \geq T(L):=e^{c L^{3 \varepsilon}}$.

In turn, at the basis of the proof of Theorem 3.4, besides the so called Peres-Winkler censoring inequality (see [41] and [37, Section 2.4]), there were two key equilibrium estimates on the behavior of (very) low temperature Ising interfaces which we now recall and which were the responsible for both the various $e^{-L^{\varepsilon}}$ error terms in $\delta_{1}, \delta_{2}$ and the constraint $\beta \gg 1$ on the inverse temperature. The latter was necessary since the techniques of [37] were based on several results of [14] on the Wulff construction, which in turn use in an essential way low temperature cluster expansion.

\subsection{Equilibrium bounds on low temperature Ising interfaces used in [37]}

The first estimate is the key for the proof of the first part of the inductive statement, namely $\mathcal{A}(L, t, \delta) \Rightarrow \mathcal{B}\left(L, t_{1}, \delta_{1}\right)$. Given the rectangle $Q_{L}$ write it as the union of two overlapping rectangles, each of which is a suitable vertical translate of the rectangle $R_{L}$ (see Figure 3). Call the lowest rectangle $B$ and the highest one $A$. 


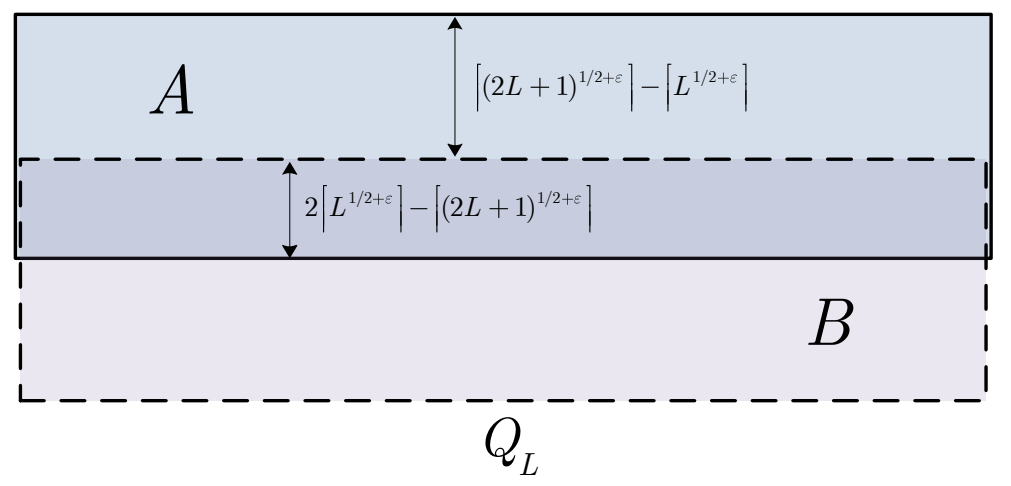

Fig. 3. The box $Q_{L}$ and its covering with the rectangles $A, B$.

Lemma 3.6 (see [37, Claim 3.6]). There exists $c=c(\beta, \varepsilon)>0$ such that

$$
\sum_{x \in B^{c}} \mathbf{E}\left(\pi^{\tau}\left(\sigma_{x}=+\right)-\pi^{\tau,-}\left(\sigma_{x}=+\right)\right) \leq e^{-c L^{2 \varepsilon}}
$$

where $\pi^{\tau,-}$ denotes the Gibbs measure in $Q_{L}$ with minus boundary conditions on its lowest side and $\tau$ on the other three sides.

In turn, by suitably playing with monotonicity properties of the measure $\pi$ as a function of the boundary conditions (see the short discussion in the proof of Claim 3.6 in [37]), the proof of the lemma can be reduced to establishing the following bound.

Consider the enlarged rectangle $E_{L}$ with sides $3 L$ and $2\left\lceil(2 L+1)^{1 / 2+\varepsilon}\right\rceil$ respectively, which can be viewed as consisting of six rectangles $Q_{L}$ stacked together. Let $\pi^{(-,-,+,-)}$ be the associated Gibbs measure with $(-)$ boundary conditions on the North, East and West sides and $(+)$ on the South side. For any spin configuration $\sigma \in\{-1,+1\}^{E_{L}}$ let $\gamma=\gamma(\sigma)$ denote the unique open contour corresponding to these boundary conditions.

Lemma 3.7. For any $\beta$ large enough there exists $c=c(\beta, \varepsilon)$ such that for any $L$,

$$
\pi^{(-,-,+,-)}\left(\gamma \text { reaches height } L^{1 / 2+\varepsilon}\right) \leq e^{-c L^{2 \varepsilon}} .
$$

Notice that the height $L^{1 / 2+\varepsilon}$ is well beyond the typical $O(\sqrt{L})$ fluctuations of the interface.

The second equilibrium bound is required for the proof of the statement $\mathcal{B}\left(L, t_{1}, \delta_{1}\right)$ $\Rightarrow \mathcal{A}\left(2 L+1, t_{2}, \delta_{2}\right)$ (see [37, Section 3.2 and in particular Claim 3.10]). Here the bottom line is the following bound.

Let $\bar{R}_{L}$ consist of two copies of $R_{L}$ stacked one on top of the other. Let $\Delta \subset \partial \bar{R}_{L}$ consist of those boundary sites $x=(i, j)$ in the South border such that $|i-L / 2| \leq \frac{1}{2} L^{3 \varepsilon}$ and $j=0$. Consider the Gibbs measure $\pi_{\bar{R}_{L}}^{(-,+, \Delta)}$ on $\bar{R}_{L}$ with (-) boundary conditions on the union of the North boundary and $\Delta$ and ( + ) on the rest of $\partial \bar{R}_{L}$. Let $\Gamma_{1}$ be the event that the open contour $\gamma_{1}$ starting on the upper left corner of $\bar{R}_{L}$ ends at the left end of the interval $\Delta$ without ever crossing the vertical line at $i=L / 2$. 


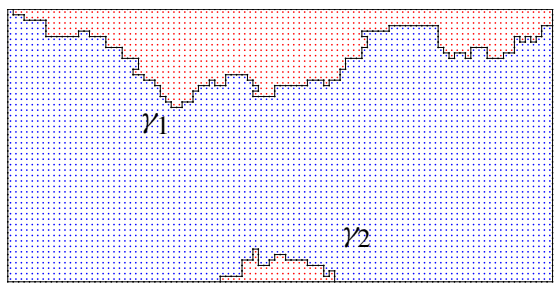

$\Delta$

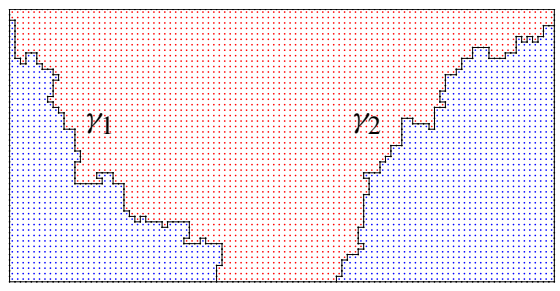

$\Delta$

Fig. 4. Two possible arrangements for the open Peierls contours in the Gibbs measure under a $(-,+, \Delta)$ boundary condition.

Lemma 3.8. For any $\beta$ large enough there exists $c=c(\beta, \varepsilon)$ such that for any $L$,

$$
\pi_{\bar{R}_{L}}^{(-,+, \Delta)}\left(\Gamma_{1}^{c}\right) \leq e^{-c L^{3 \varepsilon}}
$$

In the scheme envisaged in [37] the role played by the tiny extra piece of $(+)$ boundary conditions at the vertices of $\Delta$, being the main source of the $e^{c_{3} L^{3 \varepsilon}}$ factor relating the time scales $t_{2}, t_{1}$ in (3.3), is quite crucial and therefore it needs a bit of explanation.

Let us first explain why the length of $\Delta$ was chosen equal to $L^{3 \varepsilon}$. Under the boundary conditions $(-,+, \Delta)$, for any configuration there exist exactly two open Peierls contours $\gamma_{1}, \gamma_{2}$ with two possible scenarios for their endpoints (illustrated in Figure 4):

(a) $\gamma_{1}$ joins the two upper corners of $\bar{R}_{L}$ and $\gamma_{2}$ the two ends of the interval $\Delta$;

(b) $\gamma_{1}$ joins the left upper corner of $\bar{R}_{L}$ with the left boundary of $\Delta$ whereas $\gamma_{2}$ joins the right upper corner of $\bar{R}_{L}$ with the right boundary of $\Delta$.

In [37] it was shown, using a significant part of the main machinery of [14], that the ratio between the probabilities of the two cases is roughly of the form $e^{-\beta(L+|\Delta|-2 D) \tau_{\beta}(0)}$ where $D$ is the Euclidean distance between the left upper corner of $\bar{R}_{L}$ and the left boundary of $\Delta$. Clearly $D \approx L / 2-|\Delta|+O\left(L^{2 \varepsilon}\right)$ and therefore case (b) is much more likely than case (a) iff $|\Delta| \gg L^{2 \varepsilon}$. The choice $L^{3 \varepsilon}$ was clearly not optimal and just a very safe one. Once the first scenario can be neglected then the fact that $\gamma_{1}$ does not intersect the vertical line at $i=L / 2$ is quite natural (but painful to prove).

Next we sketchily explain the need of attracting the contour $\gamma_{1}$ deep down inside the rectangle $\bar{R}_{L}$.

When proving the implication $\mathcal{B}\left(L, t_{1}, \delta_{1}\right) \Rightarrow \mathcal{A}\left(2 L+1, t_{2}, \delta_{2}\right)$ we can imagine that the rectangle $R_{2 L+1}$ is written as the union of three copies of the rectangle $Q_{L}$ denoted by $Q_{L}^{\text {centr }}, Q_{L}^{\text {left }}, Q_{L}^{\text {right }}$ (see Figure 5 ).

For simplicity suppose that the boundary conditions around $R_{2 L+1}$ are the "extreme ones", namely $(-,-,+,-)$ ordered clockwise starting from the North one, and imagine starting the dynamics from all pluses.

The Peres-Winkler results allow us to e.g. first run the dynamics in the central rectangle $Q_{L}^{\text {centr }}$ for a time $t_{1}$ and then in the left and right ones for some other time lag. Thus the dynamics in $Q_{L}^{\text {centr }}$ runs with b.c. $(-,+,+,+)$ and after a time lag $t_{1}$ it will be close to 


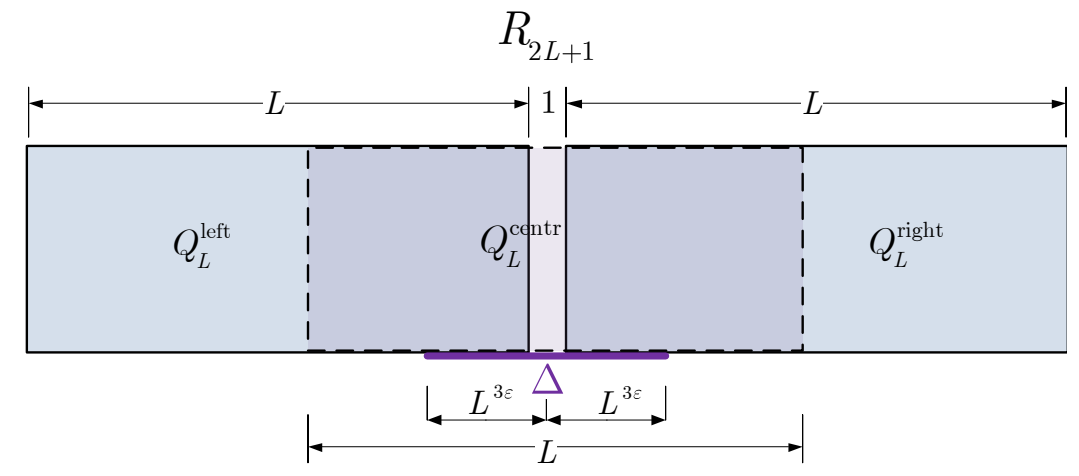

Fig. 5. The box $R_{2 L+1}$ and its covering with $Q_{L}^{\text {centr }}, Q_{L}^{\text {left }}, Q_{L}^{\text {right }}$. In bold the exceptional set $\Delta$.

the Gibbs measure $\pi_{Q_{L}^{\text {centr }}}^{(-,+,+)}$by less than $\delta_{1}$ because of $\mathcal{B}\left(L, t_{1}, \delta_{1}\right)$. The trouble is that the marginal of this measure on e.g. the East boundary of $Q_{L}^{\text {left }}$ is not dominated by $\pi_{\infty}^{-}$ because the unique open contour joining the left upper corner of $Q_{L}^{\text {centr }}$ to the right one will stay close to the upper side of $Q_{L}^{\text {centr }}$. Therefore we cannot use statement $\mathcal{B}\left(L, t_{1}, \delta_{1}\right)$ for the dynamics in $Q_{L}^{\text {left }}$ to force equilibrium there in another time lag $t_{1}$.

An appealing and very intuitive possible way out of this serious problem would be to run many times the dynamics in $Q_{L}^{\text {centr }}$ until a large deviation forces the open contour to go below and to the left of the East side of $Q_{L}^{\text {left }}$. Since the probability of this fluctuation is $O\left(\exp \left(-c L^{2 \varepsilon}\right)\right)$ it would be enough to wait $O\left(\exp \left(c L^{2 \varepsilon}\right) t_{1}\right)$ runs. However a rigorous implementation of this idea is far from trivial and in [37] the solution was another one, less natural but much easier to carry out.

If one, by brute force, flips the boundary conditions inside the interval $\Delta$ on the South side of $Q_{L}^{\text {centr }}$ to $(-)$, the mixing time of the dynamics cannot change by more than $\exp (c(\beta)|\Delta|)($ see [37, Section 2.5] for more details). Once the boundary conditions have been flipped, then, thanks to (3.8), the contours in $Q_{L}^{\text {centr }}$ will follow scenario (b) above and the resulting distribution over the East boundary of $Q_{L}^{\text {left }}$ will now be dominated by the minus phase $\pi_{\infty}^{-}$allowing another application of the inductive statement $\mathcal{B}\left(L, t_{1}, \delta_{1}\right)$ to $Q_{L}^{\text {left }}$ and $Q_{L}^{\text {right }}$.

\section{A new recursive scheme}

In this section we modify the recursion scheme of [37] and, modulo two equilibrium estimates very similar to Lemmas 3.7 and 3.8, we prove Theorem 1 . We begin by fixing some notation.

Let $N \in \mathbb{N}$ be a large integer, let $L=L_{N}=2^{N}-1$ and choose $N_{0}$ to be the smallest integer such that $L_{N_{0}}:=2^{N_{0}}-1 \geq\left\lfloor(\log L)^{3}\right\rfloor$. In our recursion $N_{0}$ and $N$ will represent the initial and final scales respectively. To any intermediate scale $n \in\left[N_{0}, N\right]$ we associate a length scale $L_{n}=2^{n}-1$. We also define the rectangles $R_{n}, Q_{n}$ to have sides 
(parallel to the coordinate axes) of length $\left(L_{n}, \kappa_{N} \sqrt{L_{n}}\right)$ and $\left(L_{n}, \kappa_{N} \sqrt{L_{n+1}}\right)$ respectively where $\kappa_{N}=\sqrt{\kappa N}=O\left((\log L)^{1 / 2}\right)$ and $\kappa$ is a positive constant that later will be chosen large enough depending on $\beta$. Thus the very definition of the rectangles depends on the final scale (which was not the case for the rectangles of the previous section). It is worth noticing that $L_{n} \gg \kappa_{N} \sqrt{L_{n+1}}$ for any $n \in\left[N_{0}, N\right]$. Finally, for any $n \in\left[N_{0}, N\right]$, we define the statements $\mathcal{A}\left(L_{n}, t, \delta\right)$ and $\mathcal{B}\left(L_{n}, t, \delta\right)$ as in Definition 3.2.

Having fixed the basic notation we can formulate our inductive scheme as follows. We repeat the result on the starting point for completeness, despite it being completely obvious after Proposition 3.3 and the remark after it.

Proposition 4.1 (The starting point). For every $\beta$ there exists $c=c(\beta)$ such that for every $n \in\left[N_{0}, N\right]$ the statements $\mathcal{A}\left(L_{n}, t, e^{-t e^{-c \kappa_{N} \sqrt{L_{n}}}}\right)$ and $\mathcal{B}\left(L_{n}, t, e^{-t e^{-c \kappa_{N} \sqrt{L_{n}}}}\right)$ hold.

Theorem 4.2 (The inductive step). There exist constants $c_{1}, c_{2}, c_{3}$ and for every $\beta>\beta_{c}$ there exists $\kappa_{0}$ such that for any $\kappa \geq \kappa_{0}$, any $N$ large enough and any $n \in\left[N_{0}, N\right]$,

$$
\mathcal{A}\left(L_{n}, t_{n}, \delta_{n}\right) \Rightarrow \mathcal{B}\left(L_{n}, t_{n}^{\prime}, \delta_{n}^{\prime}\right) \Rightarrow \mathcal{A}\left(L_{n+1}, t_{n+1}, \delta_{n+1}\right)
$$

where

$$
\begin{aligned}
\delta_{n}^{\prime} & =c_{1}\left(\delta_{n}+e^{-c_{2} \kappa_{N}^{2}}+L_{n}^{2} e^{-c_{2} \log t_{n}}\right), \quad t_{n}^{\prime}=2 t_{n}, \\
\delta_{n+1} & =c_{3}\left(\delta_{n}+e^{-c_{2} \kappa_{N}^{2}}+L_{n}^{2} e^{-c_{2} \log t_{n}}\right), \quad t_{n+1}=e^{c_{3} \kappa_{N}^{2}} t_{n} .
\end{aligned}
$$

Corollary 4.3 (Solving for the final scale). In the same setting of Theorem 4.2 there exists $c>0$ such that, if $t_{N}:=e^{c \kappa N^{2}}$ and $\delta_{N}:=c e^{-c^{-1} \kappa N}$, then for any $N \in \mathbb{N}$ large enough statement $\mathcal{A}\left(L_{N}, t_{N}, \delta_{N}\right)$ holds.

Proof of the Corollary. Choose $t_{N_{0}}=e^{c^{\prime} \kappa N^{2}}$ for some $c^{\prime}>0$. Thanks to Proposition 4.1, for any $\beta \geq 0$ it is possible to choose $c^{\prime}=c^{\prime}(\beta)$ in such a way that $\mathcal{A}\left(L_{N_{0}}, t_{N_{0}}, \delta_{N_{0}}\right)$ holds with $\delta_{N_{0}}=e^{-c^{\prime} \kappa N^{2} / 2}$. Theorem 4.2 immediately implies (use $t_{n} \geq t_{N_{0}}$ ) that $t_{N} \leq e^{c \kappa N^{2}}$ and $\delta_{N} \leq c e^{-c^{-1} \kappa N}$ for some other constant $c$.

Once Corollary 4.3 is proved, Theorem 1 and its corollaries (Corollaries 2 and 4) follow by exactly the same arguments envisaged in [37] for the analogous results (namely, for the proof of Theorem 1 given Corollary 4.3 see [10, Section 4.1]; the analog of Corollary 2 is [37, Corollary 1.7] and the analog of Corollary 4 is [37, Corollary 1.10]).

In turn the proof of Theorem 4.2 follows step by step the proof of Theorem 3.4 in [37] once we assume two key bounds on Ising interfaces that we state below.

\subsection{Two key equilibrium estimates for the new recursion}

Consider a rectangle with boundary conditions that are identically $(+)$ on the South boundary and (-) elsewhere. The following proposition addresses a large deviation esti- 


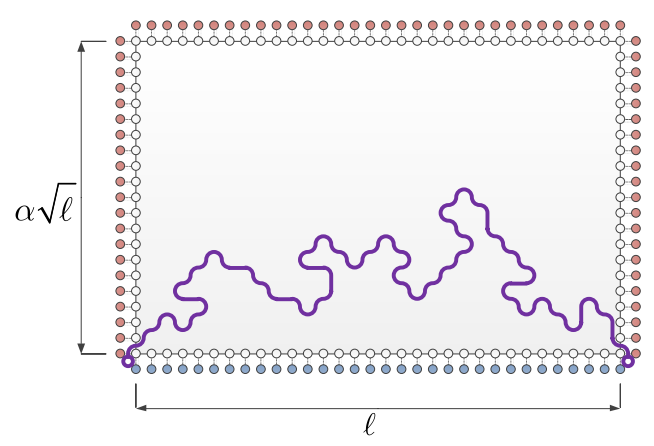

Fig. 6. Large deviation estimate for vertical fluctuations of the open Peierls contour under $(-,-,+,-)$ b.c., established in Proposition 4.4.

mate for the vertical fluctuations of the unique open contour in this setting, as illustrated in Figure 6.

Proposition 4.4. Let $R$ be a rectangle of dimensions $\ell \times \alpha \sqrt{\ell}$ with $1<\alpha \leq \sqrt{\ell}$ and let $\pi_{R}^{(-,-,+,-)}$be the corresponding Ising Gibbs measure with $(-,-,+,-)$ordered clockwise starting from the North side. Let $\lambda=\lambda(\sigma)$ denote the unique open Peierls contour of the spin configuration $\sigma \in \Omega_{R}$. Then for any $\beta>\beta_{c}$ there exist constants $c_{1}, c_{2}>0$ depending only on $\beta$ such that for any $0<\delta<1$ and $\alpha, \ell$ as above

$$
\pi_{R}^{(-,-,+,-)}(\sigma: \lambda(\sigma) \text { reaches height } \delta \alpha \sqrt{\ell}) \leq \ell^{c_{1}} e^{-c_{2}(\delta \alpha)^{2}} .
$$

Remark. In the proof of the statement $\mathcal{A}\left(L_{n}, t_{n}, \delta_{n}\right) \Rightarrow \mathcal{B}\left(L_{n}, t_{n}^{\prime}, \delta_{n}^{\prime}\right)$ for $n \in\left[N_{0}, N\right]$, the above proposition is used with $\ell=3 L_{n}, \alpha=\sqrt{\ell} / 3$ and $\delta=\sqrt{3} \kappa_{N} / \sqrt{\ell}$ (cf. [37, Section 3.1, in particular the proof of Claim 3.6]). Thus for $\kappa$ large enough depending on $\beta$ and for every $n \in\left[N_{0}, N\right]$ the r.h.s. of (4.4) is quite small.

The second equilibrium bound that is needed can be formulated as follows. Mark the rectangle $R$ as given above by the corners $\left(x, y, y^{\prime}, x^{\prime}\right)$ clockwise starting from the Northwest corner. Consider the Ising Gibbs measure on $R$ with the following b.c.:

(i) -1 on the North boundary and on an interval $\Delta$ of length $s \alpha^{2}$ belonging to the South boundary and centered around its midpoint;

(ii) +1 elsewhere.

We refer to these boundary conditions as the b.c. $(-,+, \Delta)$ and let $u$ and $v$ denote the West and East endpoints of the interval $\Delta$ centered on the South border.

In this new setting we aim to show that with high probability in the random-line representation there are two open contours $\lambda_{1}$ and $\lambda_{2}$ with $\delta \lambda_{1}=\{x, u\}$ and $\delta \lambda_{2}=\{y, v\}$ and such that $\lambda_{1}$ (resp. $\lambda_{2}$ ) lies entirely in the left (resp. right) half of $R$, as shown in Figure 7 (recall the discussion following Lemma 3.8 for the role of this event in the inductive scheme). This is established by the next proposition. 


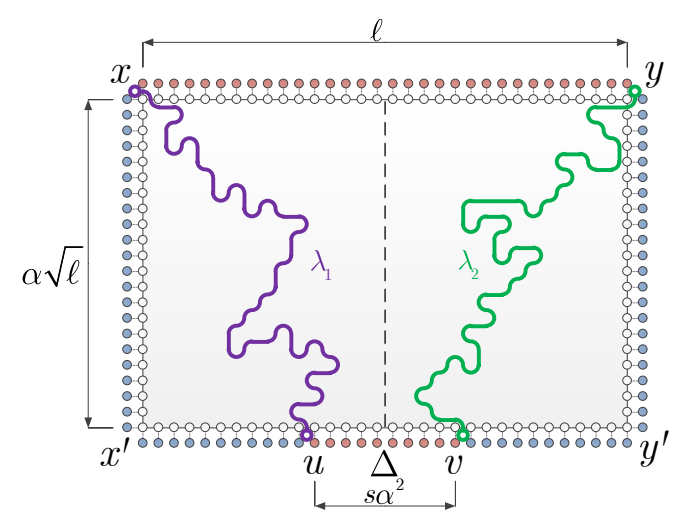

Fig. 7. Open Peierls contours confined to the left and right halves of the rectangle $R$ under $(-,+, \Delta)$ b.c., addressed by the equilibrium estimate of Proposition 4.5 .

Proposition 4.5. For any $\beta>\beta_{c}$ there exist $c_{1}, c_{2}, s_{0}>0$ depending only on $\beta$ so that the following holds. Let $R$ be a rectangle of size $\ell \times \alpha \sqrt{\ell}$ with $1<\alpha<\left(1 / s_{0}\right) \sqrt{\ell}$ and let $\Delta=[u, v]$ be an interval of length s $\alpha^{2}$ centered on the South border for some $s \geq s_{0}$. Let $\mathcal{V}$ denote the event that there are two open Peierls contours confined to the left and right halves of $R$ and connecting the top corners with $u, v$. Then

$$
\pi^{(-,+, \Delta)}\left(\mathcal{V}^{c}\right) \leq \ell^{c_{1}} e^{-c_{2} \alpha^{2}} .
$$

Remark. In the proof of the statement $\mathcal{B}\left(L_{n}, t_{n}^{\prime}, \delta_{n}^{\prime}\right) \Rightarrow \mathcal{A}\left(L_{n+1}, t_{n+1}, \delta_{n+1}\right)$ the above proposition is invoked with a choice of $\ell=L_{n}, n \in\left[N_{0}, N\right]$, and $\alpha=2 \sqrt{2} \kappa_{N}$ (cf. [37, Section 3.2 and in particular the steps leading to (3.32)]), so that $\alpha=o(\sqrt{\ell})$; also, the r.h.s. of (4.5) is always very small provided that the constant $\kappa$ is chosen to be large enough.

\subsection{Recursive analysis and quasi-polynomial bounds}

Using the above described recursive scheme, our bound on the mixing time of the dynamics is essentially optimal, even if one assumes the sharpest possible random walk-like estimates on open contours. In some sense the recursion has an intrinsic limitation which, apparently, prevents the possibility of going beyond the quasi-polynomial bound. In our approach, the proof of the inductive statement $\mathcal{A}\left(L_{n}, t_{n}, \delta_{n}\right) \Rightarrow \mathcal{A}\left(L_{n+1}, t_{n+1}, \delta_{n+1}\right)$ is in fact split into two steps: (i) the proof of $\mathcal{A}\left(L_{n}, t_{n}, \delta_{n}\right) \Rightarrow \mathcal{B}\left(L_{n}, t_{n}^{\prime}, \delta_{n}^{\prime}\right)$ and (ii) that of $\mathcal{B}\left(L_{n}, t_{n}^{\prime}, \delta_{n}^{\prime}\right) \Rightarrow \mathcal{A}\left(L_{n+1}, t_{n+1}, \delta_{n+1}\right)$. These two steps unfortunately impose conflicting requirements on the shape of the rectangles $R_{n}$. The first step requires the height $H_{n}$ of $R_{n}$ to be so large that with high probability an open contour with end-points at e.g. the lowest corners of $R_{n}$ does not reach a height of $H_{n} / 2$. That forces $H_{n}$ to be a large constant times $\sqrt{L_{n} \log L_{n}}$. On the other hand, in the proof of the second step, one asks the contour to "climb" (or to "descend") a height of order $H_{n}$, an event which due to the above restriction on the size of $H_{n}$ has probability poly $\left(L^{-1}\right)$. Since, roughly speaking, the cost 
relating the two times $t_{n}$ and $t_{n+1}$ is the inverse of the probability of such a deviation, one gets the poly $(L)$ loss in the mixing time for each iteration.

At a higher level, what is really missing in our approach is the fact that, in reality, contours evolve because they feel a drift due to their curvature and not because they exploit anomalously large deviations like the one required in the proof of step (ii).

In $[9,10]$ this fact was implemented and made rigorous for e.g. the one-dimensional solid-on-solid model using Wilson's method [50] which, by exploiting curvature of the contour, provides a quite detailed control over the mixing time. Unfortunately, something comparable with Wilson's method is not available for the full Ising model.

We end this section by noting the advantage of using duality over cluster expansion in the analysis of the equilibrium properties of contours. Duality techniques allow one to use in a natural way very useful monotonicity properties (see e.g. Lemma 2.2) which are essentially a consequence of the GKS inequalities. This feature is missing in the cluster expansion approach. Another disadvantage of the cluster expansion approach is the requirement that $\beta$ is large enough and the presence of spurious multiplicative error terms of the type $\exp \left(-(\log L)^{c}\right)$ or even $\exp \left(-L^{\varepsilon}\right)$ when lower bounding the probability of some events (cf. for instance [37, Lemma A.6] or [37, Eq. (A15)]).

\section{Equilibrium crossing probabilities for the infinite strip}

In this section we study the behavior of the unique open contour in the infinite strip with boundary conditions $(+)$ in the upper half-plane and $(-)$ in the lower half-plane. Deriving sharp estimates for the probability that this contour is confined to the upper halfplane, as well as a large deviation estimate for the its vertical fluctuations, will later serve as a key element in the proofs of Propositions 4.4 and 4.5. The analysis in this section hinges on the duality tools developed in $[43,44]$, which enable us to characterize the Ising interfaces for any $\beta>\beta_{c}$. By using this machinery together with some additional ideas we establish various properties of the contours, roughly analogous to Brownian bridges with logarithmic "decorations".

Remark. We thank an anonymous referee for sketching an argument to obtain Theorems 5.1 and 5.3 below using the methods recently developed e.g. in [7,8]. We present our original argument which is more self-contained (and in a sense more elementary) and in addition includes a multi-scale analysis which we believe is of independent interest. The alternative approach, which also uses duality techniques in a crucial way, is based on the fact that contours can be decomposed into "irreducible" components whose sizes have exponential tails. Modulo a non-trivial amount of details, one can then treat the contour as a random walk with almost bounded steps, at which point deriving the two theorems would be standard.

For $S \subset \mathbb{Z}^{2}$ define the boundary condition $\eta \in\{ \pm 1\}^{\mathbb{Z}^{2} \backslash S}$ to be

$$
\eta(x, y)= \begin{cases}-1, & y>0 \\ +1, & y \leq 0\end{cases}
$$




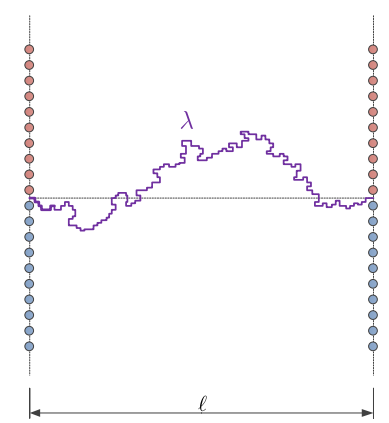

Fig. 8. Infinite strip with mixed b.c. and its unique open contour.

We focus on the case where $S$ is the infinite strip of width $\ell$,

$$
S=\{1, \ldots, \ell\} \times \mathbb{Z},
$$

whereby the above b.c. $\eta$ gives rise to a unique open contour $\lambda$ connecting the dual vertices $\{1 / 2,1 / 2\},\{\ell+1 / 2,1 / 2\}$ in $S^{*}$ (see Figure 8 ). Such contours have been intensively studied and the scaling limit of $\lambda$ is known to be the 1D Brownian bridge between these two points [23], while our proof requires more quantitative estimates. Tight large deviation estimates for vertical fluctuations of $\lambda$ are necessary in several places in our proof. This will be established by Theorem 5.3 below (in a slightly more general setting) via an argument akin to those used for controlling the deviations of the Brownian bridge, yet carried out within the duality framework of $[43,44]$.

Significantly more delicate is the crucial estimate of obtaining a lower bound on the probability that $\lambda$ is contained in the upper half-plane. The Brownian bridge heuristic suggests that this event holds with probability proportional to $1 / \ell$, and as the following theorem confirms this is indeed the case.

Theorem 5.1. Let $S$ be the infinite strip of width $\ell$ with b.c. $\eta$ as given in (5.1), (5.2). For an Ising configuration $\sigma$ on $S$ let $\lambda=\lambda(\sigma)$ be its unique open contour in the dual lattice $S^{*}($ i.e. $\delta \lambda=\{(1 / 2,1 / 2),(\ell+1 / 2,1 / 2)\})$. For $i \in \mathbb{Z}$ let $H_{i}^{*}=\{1 / 2, \ldots, \ell+1 / 2\} \times$ $\{i+1 / 2\}$ be the vertices forming the $i$-th horizontal level of $S^{*}$. Then for every $\ell$,

$$
c / \ell \leq \pi_{S}^{\eta}\left(\sigma: \lambda(\sigma) \text { stays above } H_{-1}^{*}\right) \leq C / \ell,
$$

where $c, C>0$ are constants that depend only on $\beta$.

As an immediate consequence we obtain the following lower bound on the spin-spin correlation at high temperature for two points on the horizontal boundary of the half-strip $S^{+}=\{1 / 2, \ldots, \ell+1 / 2\} \times \mathbb{Z}^{+*}$, which to our knowledge was previously unknown.

Corollary 5.2. Let $u=(1 / 2,1 / 2)$ and $v=(\ell+1 / 2,1 / 2)$. For every $\beta>\beta_{c}$ there exist constants $c, c^{\prime}$ such that

$$
\frac{c}{\ell^{3 / 2}} e^{-\tau_{\beta}(u-v)} \leq \pi_{S^{+}}^{*}\left(\sigma_{u} \sigma_{v}\right) \leq \frac{c^{\prime}}{\ell^{3 / 2}} e^{-\tau_{\beta}(u-v)} .
$$


Note that the above corollary also extends to other geometries, for instance rectangles with a wide range of aspect ratios where $u, v$ correspond to the upper corners. We postpone the proof of Theorem 5.1 and Corollary 5.2 in order to first obtain several of the ingredients that it would require, the first of which being the aforementioned large deviation inequality for the open contour in the infinite strip $S$.

Theorem 5.3. Let $\bar{S}=\bar{S}(a, b)$ be the infinite strip $\{1, \ldots, \ell\} \times \mathbb{Z}$ excluding the horizontal slits $\{1, \ldots, a\} \times\{0,1\}$ and $\{b, \ldots, \ell\} \times\{0,1\}$ for $0 \leq a<b \leq \ell+1$ with b.c. $\eta$ as defined in (5.1). For an Ising configuration $\sigma$ on $\bar{S}$ let $\lambda=\lambda(\sigma)$ be its unique open contour in the dual $\bar{S}^{*}$, and for $i \in \mathbb{Z}$ let $H_{i}^{*}=\{1 / 2, \ldots, \ell+1 / 2\} \times\{i+1 / 2\}$. Then there exists some constant $C(\beta)>0$ such that for any $\ell$ the following holds:

$$
\begin{aligned}
\pi \frac{\eta}{\bar{S}}\left(\sigma: \lambda(\sigma) \text { reaches } H_{x \sqrt{\ell}}^{*}\right) & \leq C \exp \left(-\kappa_{\beta} x^{2}\right) \quad \text { for all } x \leq \sqrt{\ell}, \\
\pi_{\bar{S}}^{\eta}\left(\sigma: \lambda(\sigma) \text { reaches } H_{h}^{*}\right) & \leq C \exp \left(-\kappa_{\beta} h\right) \quad \text { for all } h \geq \ell,
\end{aligned}
$$

where $\kappa_{\beta}>0$ is the constant in the sharp triangle inequality of the surface tension $\tau_{\beta}$.

Remark. It is fairly straightforward to establish upper bounds as above with an extra prefactor of order $|b-a|$ (see e.g. the first inequality in (5.12)). Eliminating this spurious prefactor requires a delicate multi-scale analysis.

Proof of Theorem 5.3. In what follows we will prove the following inequality, which is a stronger form of the required large deviation estimates: For some $C=C(\beta)>0$,

$$
\pi_{\bar{S}}^{\eta}\left(\sigma: \lambda(\sigma) \text { reaches } H_{h}^{*}\right) \leq C e^{-\kappa \beta\left(\frac{h^{2}}{b-a-1} \wedge h\right)} \quad \text { for any } h>0
$$

(we may clearly assume that $b>a+1$, as otherwise the unique open contour is trivial). Indeed, the above probability estimate is clearly increasing in the value of $b-a$, which in turn is guaranteed to be at most $\ell+1$ (reflecting the bounds in the proposition). Notice that by choosing $C$ to be appropriately large we need only consider $h \geq \sqrt{b-a}$.

Fix some large cutoff height $n \geq(h \vee \ell)^{2}$ and let

$$
\bar{S}_{n}=\bar{S} \cap(\mathbb{Z} \times\{-n, \ldots, n\})
$$

be the strip $\bar{S}$ truncated at $\pm n$ with boundary conditions analogous to $\eta$, i.e. negative on the upper half-plane and positive elsewhere. Due to the uniqueness of the Gibbs measure on $\bar{S}$, the probabilities we seek to bound are obtained as a limit of the corresponding ones for $\bar{S}_{n}$ as $n \rightarrow \infty$. Further let $u=(a+1 / 2,1 / 2)$ and $v=(b-1 / 2,1 / 2)$ denote the endpoints of the unique open contour in $\bar{S}_{n}^{*}$. Define the height of this open contour $\lambda \subset \bar{S}_{n}^{*}$ at the horizontal coordinate $x \in\{1 / 2, \ldots, \ell+1 / 2\}$ to be

$$
\operatorname{ht}(\lambda, x)=\max \{y:(x, y) \in \lambda\} .
$$

The main effort in the proof will be devoted to the analysis of the vertical fluctuations of the contour $\lambda$ within the inner strip with $x$-coordinates $\{a+1 / 2, \ldots, b-1 / 2\}$. It is the case that large vertical fluctuations in the margins (i.e. large values of $\operatorname{ht}(\lambda, x)$ for $x<a$ or $x>b$ ) are far more unlikely and can be estimated via standard properties of the surface 
tension. To control the delicate fluctuations of $\mathrm{ht}(\lambda, x)$ for $a<x<b$ we will apply a multiscale approach, repeatedly bounding the deviations at the horizontal midpoints in a nested dyadic partition of the interval between $u$ and $v$.

The first step in the proof is to bound the event that the contour includes a given point $w=(x, h) \in \bar{S}^{*}$ in terms of its coordinates $h$ and $x$. First notice that by (2.8),

$$
\pi_{\bar{S}_{n}}^{\eta}(\sigma: w \in \lambda(\sigma))=\left[\sum_{\substack{\lambda: \delta \lambda=\{u, v\} \\ w \in \lambda}} q_{\bar{S}_{n}^{*}}(\lambda)\right] /\left[\sum_{\lambda: \delta \lambda=\{u, v\}} q_{\bar{S}_{n}^{*}}(\lambda)\right] .
$$

Consider the numerator in the last expression: Corollary 2.5 implies that

$$
\sum_{\substack{\lambda: \delta \lambda=\{u, v\} \\ w \in \lambda}} q_{\bar{S}_{n}^{*}}(\lambda) \leq \pi_{\bar{S}_{n}^{*}}^{*}\left(\sigma_{u} \sigma_{w}\right) \pi_{\bar{S}^{*}}^{*}\left(\sigma_{v} \sigma_{w}\right)
$$

and together with Lemma 2.1 we deduce that for some $c_{0}=c_{0}(\beta)>0$,

$$
\sum_{\substack{\lambda: \delta \lambda=\{u, v\} \\ w \in \lambda}} q_{\bar{S}_{n}^{*}}(\lambda) \leq \frac{c_{0}}{\sqrt{|u-w||v-w|}} \exp \left(-\tau_{\beta}(u-w)-\tau_{\beta}(v-w)\right) .
$$

To estimate the denominator in (5.4) recall (2.7) according to which

$$
\sum_{\lambda: \delta \lambda=\{u, v\}} q_{\bar{S}_{n}^{*}}(\lambda)=\pi_{\bar{S}_{n}^{*}}^{*}\left(\sigma_{u} \sigma_{v}\right) .
$$

As it follows from GKS that decreasing our domain can only decrease the spin-spin correlations, letting $S_{n}=\{a, \ldots, b\} \times\{-n, \ldots, n\}$ (i.e. $S_{n}$ is the result of "pushing" the West and East boundaries of $\bar{S}_{n}$ to $a$ and $b$ resp.) we have

$$
\pi_{\bar{S}_{n}^{*}}^{*}\left(\sigma_{u} \sigma_{v}\right) \geq \pi_{S_{n}^{*}}^{*}\left(\sigma_{u} \sigma_{v}\right) .
$$

By (2.3) there exists some $c_{1}=c_{1}(\beta)>0$ such that the spin-spin correlation between $u, v$ in the dual to the infinite $\operatorname{strip} S=\{a, \ldots, b\} \times \mathbb{Z}$ is

$$
\pi_{S^{*}}^{*}\left(\sigma_{u} \sigma_{v}\right)=\frac{c_{1}+o(1)}{\sqrt{|u-v|}} \exp \left(-\tau_{\beta}(|u-v|)\right),
$$

where the $o(1)$-term tends to 0 as $|u-v| \rightarrow \infty$. Due to the strong spatial mixing properties of the high temperature region $\beta^{*}<\beta_{c}$, the value of $\pi_{S_{n}^{*}}^{*}\left(\sigma_{u} \sigma_{v}\right)$ converges to the above r.h.s. exponentially fast in $n$. Already for $n \geq \ell^{2}$ we could absorb the error in the constant $c_{1}$ and deduce that for some $c_{1}^{\prime}(\beta)>0$,

$$
\sum_{\lambda: \delta \lambda=\{u, v\}} q_{\bar{S}_{n}^{*}}(\lambda) \geq \frac{c_{1}^{\prime}}{\sqrt{|u-v|}} \exp \left(-\tau_{\beta}(|u-v|)\right) .
$$

By combining (5.4) with (5.5) and (5.6) we conclude that for some $c_{2}=c_{2}(\beta)>0$,

$$
\pi_{\bar{S}_{n}}^{\eta}(\sigma: w \in \lambda(\sigma)) \leq \frac{c_{2} \sqrt{|u-v|}}{\sqrt{|u-w||v-w|}} \exp \left(-\tau_{\beta}(u-w)-\tau_{\beta}(v-w)+\tau_{\beta}(u-v)\right) .
$$


At the same time, by the sharp triangle inequality property (2.2) of the surface tension,

$$
\tau_{\beta}(u-w)+\tau_{\beta}(v-w) \geq \tau_{\beta}(u-v)+\kappa_{\beta}(|w-v|+|u-w|-|u-v|) .
$$

Recalling that $w$ is at height $h$ it is easy to verify that

$$
|w-v|+|u-w|-|u-v| \geq \frac{4 h^{2}}{\sqrt{|u-v|^{2}+4 h^{2}}+|u-v|} .
$$

Set $\xi=4 /(1+\sqrt{5})>6 / 5$ and now observe that whenever $h^{2} \leq|u-v|^{2}$ the last expression is at least $\xi h^{2} /|u-v|$, and otherwise it is at least $\xi h$. Using this bound for the r.h.s. of (5.8) now allows us to produce the following bound out of (5.7):

$$
\pi_{\bar{S}_{n}}^{\eta}(\sigma: w \in \lambda(\sigma)) \leq \frac{c_{2} \sqrt{|u-v|}}{\sqrt{|u-w||v-w|}} \exp \left(-\frac{6}{5} \kappa_{\beta}\left(\frac{h^{2}}{|u-v|} \wedge h\right)\right) .
$$

Straightforward applications of the above bounds will now yield the required bounds on the height of $\lambda$ along the margins $x \leq a+1 / 2$ and $x \geq b-1 / 2$ as well as whenever $b-a$ is uniformly bounded. Indeed, by symmetry we may assume without loss of generality that $x \leq a+1 / 2$ and note that in this case $w=(x, h)$ satisfies $|w-v| \geq|u-v|$. Applying (5.7) combined with the sharp triangle inequality as in (5.8) we get

$$
\pi_{\bar{S}_{n}}^{\eta}(\sigma: w \in \lambda(\sigma)) \leq \frac{c_{2}}{\sqrt{|u-w|}} \exp \left(-\kappa_{\beta}|u-w|\right)
$$

Summing the last expression over all $w=(x, y)$ with $x \leq a+1 / 2$ and $y \geq h$ gives

$$
\pi \frac{\eta}{\bar{S}_{n}}(\sigma: \operatorname{ht}(\lambda(\sigma), x) \geq h \text { for some } x \leq a+1 / 2) \leq C_{1} e^{-\kappa_{\beta} h}
$$

for some $C_{1}=C_{1}(\beta)>0$, and analogously

$$
\pi_{\bar{S}_{n}}^{\eta}(\sigma: \operatorname{ht}(\lambda(\sigma), x) \geq h \text { for some } x \geq b-1 / 2) \leq C_{1} e^{-\kappa_{\beta} h} .
$$

We now turn to the main task of bounding the vertical fluctuations of $\lambda$ along the interval $(a+1 / 2, b-1 / 2)$. First observe that (5.9) immediately provides the bound we seek ((5.3)) in the special case where $|u-v|=O(1)$ (with an implicit constant that may depend on $\beta)$ : In that case a simple union bound over $w=(x, h)$ for $x \in(a+1 / 2, b-1 / 2)$ yields

$$
\begin{aligned}
& \pi_{\bar{S}_{n}}^{\eta}(\sigma: \operatorname{ht}(\lambda(\sigma), x) \geq h \text { for some } a+1 / 2<x<b-1 / 2) \\
& \quad \leq c_{2}|u-v| \exp \left(-\frac{6}{5} \kappa_{\beta}\left(\frac{h^{2}}{|u-v|} \wedge h\right)\right) \leq C_{2} \exp \left(-\kappa_{\beta}\left(\frac{h^{2}}{|u-v|} \wedge h\right)\right),
\end{aligned}
$$

where $C_{2}(\beta)>0$ incorporates the uniform bound on $|u-v|$. Combined with (5.10) and (5.11), this proves the bound in (5.3) when $|u-v|=O(1)$.

Let $M \geq 2$ be some fixed integer whose value will depend only on $\beta$ and will be specified later. Justified by the above argument, assume without loss of generality that

$$
|u-v| \geq M^{2} \quad \text { and } \quad \exp \left(\frac{1}{10} \kappa_{\beta}|u-v|^{1 / 4}\right) \geq|u-v| .
$$


We claim that this in turn narrows our attention to proving (5.3) for $h$ satisfying

$$
M|u-v| \leq h^{2} \leq \frac{1}{2}|u-v|^{5 / 4} .
$$

To see this recall first that the lower bound on $h$ is justified by selecting a suitably large constant $C(\beta)$ in (5.3). For the upper bound, note that if $h^{2}>\frac{1}{2}|u-v|^{5 / 4}$ (in which case $\frac{h^{2}}{|u-v|}>\frac{1}{2}|u-v|^{1 / 4}$ whereas $h>\frac{1}{\sqrt{2}}|u-v|^{5 / 8}$ ) then (5.13) implies that $|u-v|$ is at most $\exp \left(\frac{\kappa_{\beta}}{5}\left(\frac{h^{2}}{|u-v|} \wedge h\right)\right)$, and hence (5.3) follows from a union bound over $x \in$ $(a+1 / 2, b-1 / 2)$ as in (5.12).

Consider the event whereby the contour $\lambda$ visits a point $w \in \bar{S}_{n}$ given by

$$
w=(x, y) \quad \text { for some } x \in\left(a+\frac{1}{M}|u-v|, b-\frac{1}{M}|u-v|\right) \text { and } y \geq h .
$$

Clearly $\sqrt{|u-w||v-w|} \geq \frac{1}{M}|u-v|$ and thus we can rewrite (5.9) as

$$
\pi_{\bar{S}_{n}}^{\eta}(\sigma: w \in \lambda(\sigma)) \leq \frac{c_{3}}{\sqrt{|u-v|}} \exp \left(-\frac{6}{5} \kappa_{\beta}\left(\frac{y^{2}}{|u-v|} \wedge y\right)\right),
$$

where $c_{3}>0$ depends only on $\beta$. Summing over all possible values of $y \geq h$ we now obtain

$$
\begin{aligned}
& \pi_{\bar{S}_{n}}^{\eta}(\sigma: \operatorname{ht}(\lambda(\sigma), x) \geq h) \leq \frac{c_{3}}{\sqrt{|u-v|}}\left(\sum_{y=h}^{|u-v|} e^{-\frac{6}{5} \kappa_{\beta} \frac{y^{2}}{|u-v|}}+\sum_{y \geq(h \vee|u-v|)} e^{-\frac{6}{5} \kappa_{\beta} y}\right) \\
& \quad \leq c_{3} \sum_{z=h / \sqrt{|u-v|}}^{\sqrt{|u-v|}} e^{-\frac{6}{5} \kappa_{\beta} z^{2}}+\frac{c_{3}}{\sqrt{|u-v|}} \sum_{y \geq h} e^{-\frac{6}{5} \kappa_{\beta} y} \leq c_{3}^{\prime} e^{-\frac{6}{5} \kappa_{\beta} \frac{h^{2}}{|u-v|}}+\frac{c_{3}^{\prime}}{\sqrt{|u-v|}} e^{-\frac{6}{5} \kappa_{\beta} h} \\
& \quad \leq C_{3} \exp \left(-\frac{6}{5} \kappa_{\beta}\left(\frac{h^{2}}{|u-v|} \wedge h\right)\right)
\end{aligned}
$$

where the constant $C_{3}>0$ depends only on $\beta$.

We next wish to extend the above bound on $\operatorname{ht}(\lambda, x)$ to hold simultaneously for all $x \in(a+1 / 2, b-1 / 2)$ by means of a dyadic partition of the interval between $u$ and $v$. Set

$$
K=\left\lfloor\frac{1}{2} \log _{M}|u-v|\right\rfloor
$$

and notice that (5.13) ensures that $K \geq 1$. Define the following sequence of refinements of the interval between $u$ and $v$, indexed by $k=0, \ldots, K$. We begin with the trivial partition at level 0 ,

$$
z_{0}^{(0)}=a+1 / 2, \quad z_{1}^{(0)}=b-1 / 2,
$$

and refine level $k$ into level $k+1$ by subdividing each subinterval $\left(z_{j-1}^{(k)}, z_{j}^{(k)}\right)$ into $M$ equal parts (up to integer rounding):

$$
\begin{array}{rlrl}
z_{M j}^{(k+1)} & =z_{j}^{(k)} & \text { for } j=0, \ldots, M^{k}, \\
z_{M(j-1)+i}^{(k+1)}=\left\lfloor z_{j-1}^{(k)}+\frac{i}{M}\left(z_{j-1}^{(k)}+z_{j}^{(k)}\right)\right\rfloor+\frac{1}{2} & \text { for } i=1, \ldots, M-1 \text { and } j=1, \ldots, M^{k} .
\end{array}
$$


Observe that for all admissible $j, k$ we have

$$
M^{-k}|u-v|-2<z_{j}^{(k)}-z_{j-1}^{(k)}<M^{-k}|u-v|+2,
$$

where the additive terms account for the rounding corrections along the refinements. In particular, the expression in the lower bound on the subinterval lengths satisfies

$$
M^{-k}|u-v| \geq M^{-K}|u-v| \geq \sqrt{|u-v|}>10
$$

(as $|u-v|$ is large enough). Next, define

$$
h_{k}=M^{-k / 4} h \quad \text { for } k=0, \ldots, K,
$$

and let $\Upsilon_{j}^{(k)}$ be the event that the height of the contour at $z_{j}^{(k)}$ does not exceed $\sum_{i<k} h_{i}$ :

$$
\Upsilon_{j}^{(k)}=\left\{\sigma: \operatorname{ht}\left(\lambda(\sigma), z_{j}^{(k)}\right)<\sum_{i=0}^{k-1} h_{i}\right\} \quad \text { for } k \geq 1 \text { and } 1 \leq j<M^{k} .
$$

Recalling (5.15) and rewriting it in terms of $\Upsilon_{j}^{(k)}$ and its complement $\bar{\Upsilon}_{j}^{(k)}$ we have

$$
\pi_{\bar{S}_{n}}^{\eta}\left(\bar{\Upsilon}_{j}^{(1)}\right) \leq C_{3} \exp \left(-\frac{6}{5} \kappa_{\beta}\left(\frac{h_{0}^{2}}{z_{1}^{(0)}-z_{0}^{(0)}} \wedge h_{0}\right)\right) \quad \text { for } j=1, \ldots, M-1 .
$$

Exactly the same argument yields that for general $k, 1 \leq j \leq M^{k}$ and $1 \leq i \leq M-1$,

$$
\pi_{\bar{S}_{n}}^{\eta}\left(\bar{\Upsilon}_{M(j-1)+i}^{(k+1)}, \Upsilon_{j-1}^{(k)}, \Upsilon_{j}^{(k)}\right) \leq C_{3} \exp \left(-\frac{6}{5} \kappa_{\beta}\left(\frac{h_{k}^{2}}{z_{j}^{(k)}-z_{j-1}^{(k)}} \wedge h_{k}\right)\right) .
$$

To estimate the last expression, observe that $h_{k} /\left(z_{j}^{(k)}-z_{j-1}^{(k)}\right)$ increases with $k$ roughly as $M^{3 k / 4}$. More accurately,

$$
\begin{aligned}
\frac{h_{k}}{z_{j}^{(k)}-z_{j-1}^{(k)}} & \leq \frac{M^{k / 4} h}{M^{-k}|u-v|-2} \leq \frac{M^{3 k / 4} h}{|u-v|}\left(1+\frac{2}{M^{-k}|u-v|-2}\right) \\
& <\frac{5}{4} M^{3 k / 4} \frac{h}{|u-v|}
\end{aligned}
$$

(where we used the fact that $M^{-k}|u-v|>10$ ) and similarly

$$
\frac{h_{k}}{z_{j}^{(k)}-z_{j-1}^{(k)}} \geq \frac{M^{k / 4} h}{M^{-k}|u-v|+2}>\frac{4}{5} M^{3 k / 4} \frac{h}{|u-v|} .
$$

Our choice of $K$ and the upper bound (5.14) on $h$ enable us to derive from (5.17) that for all $k \leq K$,

$$
\frac{h_{k}}{z_{j}^{(k)}-z_{j-1}^{(k)}} \leq \frac{5}{4} M^{3 K / 4} \frac{1}{\sqrt{2}}|u-v|^{-3 / 8} \leq \frac{5}{4 \sqrt{2}}<1 .
$$


In particular, this identifies the minimizer of the exponent in the r.h.s. of (5.16) and implies that

$$
\pi{\overline{S_{n}}}_{n}^{\eta}\left(\bar{\Upsilon}_{M(j-1)+i}^{(k+1)}, \Upsilon_{j-1}^{(k)}, \Upsilon_{j}^{(k)}\right) \leq C_{3} \exp \left(-\frac{6}{5} \kappa_{\beta} \frac{h_{k}^{2}}{z_{j}^{(k)}-z_{j-1}^{(k)}}\right) .
$$

Crucially however, the lower bound (5.18) also gives

$$
\frac{h_{k}^{2}}{z_{j}^{(k)}-z_{j-1}^{(k)}} \geq \frac{4}{5} M^{3 k / 4} \frac{h}{|u-v|} M^{-k / 4} h=\frac{4}{5} M^{k / 2} \frac{h^{2}}{|u-v|} .
$$

To simplify the notation put $\rho=\frac{6}{5} \kappa_{\beta} \frac{h^{2}}{|u-v|}$ and recall that $\rho \geq \frac{6}{5} \kappa_{\beta} M$ by (5.14), hence we may take $M$ sufficiently large so $\rho$ would also be large. The combination of the above inequalities together with a union bound gives

$$
\begin{aligned}
\pi_{\bar{S}_{n}}^{\eta}\left(\bigcup_{k=1}^{K-1} \bigcup_{j} \bar{\Upsilon}_{j}^{(k)}\right) & =\pi_{\bar{S}_{n}}^{\eta}\left(\bigcup_{k=1}^{K-1} \bigcup_{i, j}\left\{\bar{\Upsilon}_{M(j-1)+i}^{(k)}, \Upsilon_{j-1}^{(k-1)}, \Upsilon_{j}^{(k-1)}\right\}\right) \\
& \leq C_{3} M e^{-\rho}+C_{3} \sum_{k=2}^{K-1} M^{k} e^{-\rho \frac{4}{5} M^{(k-1) / 2}} \leq C_{3}^{\prime} e^{-\rho}=C_{3}^{\prime} e^{\frac{6}{5} \kappa_{\beta} \frac{h^{2}}{|u-v|}},
\end{aligned}
$$

where we used that $\rho \geq 2$ and $\sqrt{M} \geq \log M$ for any sufficiently large $M$ and it is understood that $\Upsilon_{j}^{(0)}$ is the full probability space.

We have reached level $K$ at which point we wish to examine the remaining points altogether. Fix some $x \in\left(z_{j-1}^{(K-1)}, z_{j}^{(K-1)}\right)$ and let

$$
\Upsilon_{x}^{\prime}=\left\{\sigma: \operatorname{ht}(\lambda(\sigma), x)<\sum_{i=1}^{K} h_{i}\right\}
$$

As established before, $h_{K} /\left(z_{j}^{(K)}-z_{j-1}^{(K)}\right)<1$ and so

$$
\pi_{\bar{S}_{n}}^{\eta}\left(\bar{\Upsilon}_{x}^{\prime}, \Upsilon_{j-1}^{(K)}, \Upsilon_{j}^{(K)}\right) \leq C_{3} \exp \left(-\frac{6}{5} \kappa_{\beta} \frac{h_{K}^{2}}{z_{j}^{(K)}-z_{j-1}^{(K)}}\right) .
$$

On the other hand, by the definition of $K$ we have $M^{K} \geq|u-v|^{1 / 2} / M$ (with the factor of $M$ due to possible integer rounding in $K$ ) and hence

$$
\frac{h_{K}^{2}}{z_{j}^{(K)}-z_{j-1}^{(K)}} \geq \frac{4}{5} M^{K / 2} \frac{h^{2}}{|u-v|} \geq \frac{4}{5} \frac{|u-v|^{1 / 4}}{\sqrt{M}} \frac{h^{2}}{|u-v|} \geq|u-v|^{1 / 4},
$$

where the last inequality is due to the lower bound on $h^{2}$ in (5.14). It now follows from (5.13) that

$$
\exp \left(-\frac{1}{10} \kappa_{\beta} \frac{h_{K}^{2}}{z_{j}^{(K)}-z_{j-1}^{(K)}}\right) \leq \exp \left(-\frac{1}{10} \kappa_{\beta}|u-v|^{1 / 4}\right) \leq|u-v|^{-1} .
$$


Together with (5.19) this implies that

$$
\pi_{\bar{S}_{n}}^{\eta}\left(\bar{\Upsilon}_{x}^{\prime}, \Upsilon_{j-1}^{(K)}, \Upsilon_{j}^{(K)}\right) \leq \frac{C_{3}}{|u-v|} \exp \left(-\frac{11}{10} \kappa_{\beta} \frac{h^{2}}{|u-v|}\right) .
$$

Summing over at most $|u-v|$ possible choices for $x$ we may now conclude that

$$
\begin{aligned}
\pi_{\bar{S}_{n}}^{\eta}\left(\mathrm{ht}(\lambda, x) \geq \alpha_{M} h \text { for some } x \in(a+1 / 2, b\right. & -1 / 2)) \\
& \leq C_{4} \exp \left(-\frac{11}{10} \kappa_{\beta} \frac{h^{2}}{|u-v|}\right),
\end{aligned}
$$

where $\alpha_{M}=\sum_{0}^{K} M^{-i / 4}<\left(1-M^{-1 / 4}\right)^{-1}$.

Finally, by choosing $M$ to be sufficiently large we can obtain $\alpha_{M}^{2}<11 / 10$, and plugging this in (5.20) (while recalling that we are in the regime where $h^{2} /|u-v| \leq h$ due to (5.14)) concludes the proof of (5.3), as required.

Remark. The truncation argument that was used in the proof of Theorem 5.3 to reduce the problem to a finite domain is applicable in our upcoming arguments as well. Henceforth, when needed, we will thus work directly in the infinite volume setting to simplify the exposition.

We now introduce the main conceptual element in the proof of Theorem 5.1. Recall our aim is to show that the open contour in the infinite strip $S=\{1, \ldots, \ell\} \times \mathbb{Z}$ has a reasonable probability - namely of order $c / \ell$ - of remaining in the upper half-plane (i.e. above the dual line $\{(x, y): y=-1 / 2\})$.

Our approach, based on the Brownian bridge heuristics, is iterative and very much based on the intuitive picture in which the open contour really consists of two simple lines $\gamma_{1}, \gamma_{2}$, traveled at constant speed, one starting from the left boundary and moving towards the right boundary and vice versa for the second one, meeting in some intermediate point. Such a picture, which can be made more precise by progressively revealing the contour from left to right and from right to left (see Figure 9), allows hitting times kind of arguments that we now explain. Let $\tau_{j}^{(i)}(i=1,2)$ be the hitting time of either
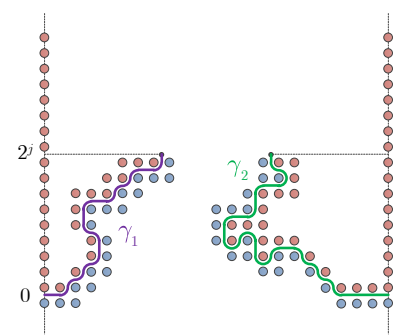

Fig. 9. The open contour in the infinite strip with mixed b.c., progressively exposed as two curves $\gamma_{1}, \gamma_{2}$ originating at its endpoints. 


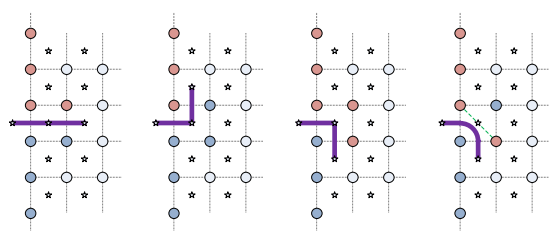

Fig. 10. Asymmetric behavior of the contour due to the global SE splitting rule.

level 0 or level $2^{j}$ for the curve $\gamma_{i}$. Then, conditioned on the event that both curves at their respective times $\tau_{j}^{(i)}$ have not yet joined and are both at level $2^{j}$, by monotonicity and symmetry, with probability at least $1 / 4$ both curves will either hit the next level $2^{j+1}$ or join together before hitting level 0 (see Claim 5.8 below for a precise formulation). Thus, with probability at least $4^{-n}$ we can force both curves to either hit level $2^{n}$ or join together before hitting level 0 . However, and that explains the heuristic bound $1 / \ell$, once the curves are at level $2^{n} \approx \sqrt{\ell}$, then with probability bounded away from 0 they will join together without hitting level 0 . In other words it is enough to force the curves to climb only $n=\frac{1}{2} \log _{2} \ell$ levels in order not to hit level 0 .

The above sketch, however, suppresses a number of technical difficulties such as the boundary conditions and the dependence between the two contours. Moreover, and in contrast to the behavior of the Brownian bridge, the law of the contour $\lambda$ is in fact asymmetric with respect to the horizontal axis. This follows from our splitting rule, which introduces a vertical bias for the contour: For instance, as illustrated in Figure 10, applying the SE splitting rule clearly has the open contour move up with probability uniformly bounded away from $1 / 2$.

To overcome this difficulty we consider the open contours formed by both the SE and the SW splitting rules, $\gamma_{\mathrm{SE}}$ and $\gamma_{\mathrm{SW}}$ resp., and examine their union $\Gamma=\gamma_{\mathrm{SE}} \cup \gamma_{\mathrm{SW}}$. Most importantly the law of their union is symmetric with respect to the horizontal axis. We will show that $\Gamma$ is essentially a "tube" of logarithmic width surrounding $\gamma_{\mathrm{SE}}$, with added "decorations" from $\gamma_{\mathrm{sw}}$ which are components of at most logarithmic diameter (and similarly if we reverse the roles of $\gamma_{\mathrm{SE}}, \gamma_{\mathrm{SW}}$ ). Up to these logarithmic corrections we may implement the heuristics of our above sketch, as stated in the following lemmas. Here and in what follows we associate with an open contour $\gamma$ going from $u$ to $v$ a unit speed parametrization $\gamma(t)$, justifying hitting-time type of events (e.g. " $\gamma$ hits the vertex $y$ prior to hitting $z "$ etc.).

Lemma 5.4. Let $\bar{S}=\bar{S}(a, b)$ and $H_{i}^{*}(i \in \mathbb{Z})$ be as in Theorem 5.3. For an Ising configuration $\sigma$ on $\bar{S}$ let $\gamma_{\mathrm{SE}}(\sigma)$ and $\gamma_{\mathrm{SW}}(\sigma)$ be the two unique open contours in $\bar{S}^{*}$ formed by the $S E$ and $S W$ splitting rules resp., i.e. going from $(a+1 / 2,1 / 2)$ to $(b-1 / 2,1 / 2)$. There exists some $C^{\star}(\beta)>0$ so that for any $h \geq 1$ the contour $\gamma_{\mathrm{SE}}\left(\right.$ resp. $\left.\gamma_{\mathrm{SW}}\right)$ hits $H_{-h-C^{\star}}^{*} \log h$ before hitting either $H_{h-C^{\star} \log h}$ or $(b-1 / 2,1 / 2)$ with probability at most $1 / 2+C^{\star} / h$.

Proof. We define the gain of a connected subset of dual edges $A$ in the infinite strip $\bar{S}$ in the interval $I \subset \mathbb{Z}$ over distance $m$, denoted by $\operatorname{gn}(A, I, m)$, to be the maximal difference in $y$-coordinates between any two points in $A$ whose $x$-coordinates are contained in $I$ and 
differ by at most $m$ :

$$
\begin{aligned}
& \operatorname{gn}(A, I, m) \\
& \quad:=\max _{x, y, x^{\prime}, y^{\prime}}\left\{\left|y-y^{\prime}\right|:\left|x-x^{\prime}\right| \leq m,(x, y) \in A,\left(x^{\prime}, y^{\prime}\right) \in A, x, x^{\prime} \in I\right\} .
\end{aligned}
$$

We define the gradient of $A$ as its gain over distance 0 . The following claim bounds the gain of $\gamma$ in a neighborhood of $a$ and $b$ :

Claim 5.5. Let $\gamma$ be the open contour with either SE or SW splitting rule in the infinite strip $\bar{S}=\bar{S}(a, b)$ of side-length $\ell$ defined in Theorem 5.3. Then for any $c>0$ there exists a constant $C_{1}^{\star}=C_{1}^{\star}(\beta, c)>0$ such that for all $1 \leq m \leq \ell$,

$$
\pi_{\bar{S}}^{\eta}\left(\operatorname{gn}(\gamma,[a-m, a+m], c \log m)>C_{1}^{\star} \log m\right) \leq C_{1}^{\star} / m .
$$

The analogous statement holds with a replaced by $b$.

Proof of Claim 5.5. Define $I=[a-m, a+m] \cap \mathbb{Z}$ and in what follows take $C_{1}^{\star} \geq c$. Further let $u=(a+1 / 2,1 / 2)$ and $v=(b-1 / 2,1 / 2)$ denote the endpoints of the open contour $\gamma$, and let $C_{1}^{\star}=C_{1}^{\star}(\beta)$ be some constant to be determined later. Define the set

$$
\Xi=\left\{\left((x, y),\left(x^{\prime}, y^{\prime}\right)\right) \in \bar{S}^{2}: x, x^{\prime} \in I,\left|x-x^{\prime}\right| \leq c \log m,\left|y-y^{\prime}\right| \geq C_{1}^{\star} \log m\right\} .
$$

If $\operatorname{gn}(\gamma, I, c \log m)>C_{1}^{\star} \log m$ then there exist $z=(x, y), z^{\prime}=\left(x^{\prime}, y^{\prime}\right) \in \gamma$ such that $\left(z, z^{\prime}\right) \in \Xi$. Taking a union bound over ordered pairs of intermediate points $z, z^{\prime} \in \Xi$ such that $z=(x, y)$ and $z^{\prime}=\left(x^{\prime}, y^{\prime}\right)$ we get

$$
\pi_{\bar{S}}^{\eta}\left(\operatorname{gn}(\gamma, I, c \log m)>C_{1}^{\star} \log m\right) \leq\left[\sum_{\left(z, z^{\prime}\right) \in \Xi} \sum_{\substack{\lambda: \delta \lambda=\{u, v\} \\ z, z^{\prime} \in \lambda}} q_{\bar{S}^{*}}(\lambda)\right] /\left[\sum_{\lambda: \delta \lambda=\{u, v\}} q_{\bar{S}^{*}}(\lambda)\right] .
$$

As we have already seen, (2.3) provides a sharp estimate for the above denominator and it remains to consider the numerator. Recall Corollary 2.5 that treated the measure of all open contours $\lambda$ in a domain $\Lambda^{*}$ that go between two endpoints $u, v$ as well as an intermediate point $z$, bounding it from above by the product of the spin-spin correlations $\pi_{\Lambda^{*}}^{*}\left(\sigma_{u} \sigma_{z}\right)$ and $\pi_{\Lambda^{*}}^{*}\left(\sigma_{v} \sigma_{z}\right)$. Following essentially the same proof, [43, Lemma 5.4] gives an analogous version of this statement for all such contours $\lambda$ going through two ordered intermediate points $z, z^{\prime}$ (that is, $\gamma$ connects $u$ to $z$, thereafter proceeds to $z^{\prime}$ and ends at $v$ ), whereby

$$
\sum_{\substack{\lambda: \delta \lambda=\{u, v\} \\ z, z^{\prime} \in \lambda}} q_{\Lambda^{*}}(\lambda) \leq \pi_{\Lambda^{*}}^{*}\left(\sigma_{u} \sigma_{z}\right) \pi_{\Lambda^{*}}^{*}\left(\sigma_{z} \sigma_{z^{\prime}}\right) \pi_{\Lambda^{*}}^{*}\left(\sigma_{z^{\prime}} \sigma_{v}\right)
$$

Therefore, Lemma 2.1 implies that

$$
\sum_{\substack{\lambda: \delta \lambda=\{u, v\} \\ z, z^{\prime} \in \lambda}} q_{\bar{S}^{*}}(\lambda) \leq \frac{C_{\beta}^{3} \exp \left(-\left[\tau_{\beta}(u-z)+\tau_{\beta}\left(z-z^{\prime}\right)+\tau_{\beta}\left(v-z^{\prime}\right)\right]\right)}{\sqrt{|u-z| \cdot\left|z-z^{\prime}\right| \cdot\left|z^{\prime}-v\right|}} .
$$


Since $\tau_{\beta}(\theta) \geq \tau_{\beta}(0)$ (see e.g. [6]) we can bound the last exponent from above by

$$
e^{-\tau_{\beta}(0)\left[|u-z|+\left|z-z^{\prime}\right|+\left|v-z^{\prime}\right|\right]} \leq e^{-\tau_{\beta}(0)\left[|u-v|+c^{\prime}\left(|y| / m+\left|y-y^{\prime}\right|\right)\right]},
$$

where $c^{\prime}>0$ is an absolute constant; indeed, the last inequality is justified by the fact that $\left|y-y^{\prime}\right|$ is at least a constant times $\left|x-x^{\prime}\right|$ (recall that $C_{1}^{*} \geq c$ ) and a similar statement holds with respect to $y / m$ compared to $|x-a|$. Since summing over $x, x^{\prime}$ amounts to a factor of $O\left(\mathrm{~m}^{2}\right)$, absorbing an additional $O(m)$ term from the sum over $y / m$ while recalling that $\left|y-y^{\prime}\right| \geq C_{1}^{\star} \log m$ now implies that

$$
\sum_{\substack{\lambda: \delta \lambda=\{u, v\} \\ z, z^{\prime} \in \lambda}} q_{\bar{S}^{*}}(\lambda) \leq C m^{-p}|u-v|^{-1 / 2} \exp \left(-\tau_{\beta}(u-v)\right),
$$

where $p$ can be made arbitrarily large by taking $C_{1}^{\star}$ large enough. In conclusion,

$$
\pi_{\bar{S}}^{\eta}\left(\operatorname{gn}(\gamma, I, c \log m)>C_{1}^{\star} \log m\right) \leq C_{1}^{\star} / m,
$$

completing the proof.

Claim 5.6. Let $\gamma_{\mathrm{SE}}$ and $\gamma_{\mathrm{SW}}$ be the open contours with the SE and SW splitting rule resp. in the infinite strip $\bar{S}=\bar{S}(a, b)$ of side-length $\ell$ defined in Theorem 5.3. Then there exists some $C_{2}^{\star}=C_{2}^{\star}(\beta)>0$ so that for all $1 \leq m \leq \ell$ with probability at least $1-C_{2}^{\star} / m$ every connected component of $\gamma_{\mathrm{SW}} \backslash \gamma_{\mathrm{SE}}$ with zero distance from $([a-m, a+m] \times \mathbb{Z}) \cap \gamma_{\mathrm{SE}}$ has diameter at most $C_{2}^{\star} \log m$.

Proof of Claim 5.6. Let $\mathcal{B}$ denote the event that there exists a connected component of $\gamma_{\mathrm{SW}} \backslash \gamma_{\mathrm{SE}}$ with zero distance from $([a-m, a+m] \times \mathbb{Z}) \cap \gamma_{\mathrm{SE}}$ and has diameter at least $C_{2}^{\star} \log m$. We begin by conditioning on $\gamma_{\mathrm{SE}}$. The contour partitions $\bar{S}$ into two sets $S_{\text {TOP }}$ and $S_{\text {вот }}$. For a set $A$ of dual edges let $V(A) \subset \mathbb{Z}^{2}$ denote the set of vertices at distance $1 / 2$ from $A$. The effect of conditioning on $\gamma_{\mathrm{SE}}$ is equivalent to conditioning that $\sigma_{U}=\eta_{U}$ where $U=V\left(\Delta\left(\gamma_{\mathrm{SE}}\right)\right)$ (recall the definition of the edge-boundary $\Delta(\cdot)$ in $\S 2$ after Lemma 2.2) and $\eta_{U} \in\{-1,+1\}^{U}$ is the configuration given by

$$
\eta_{u}= \begin{cases}-1, & u \in S_{\mathrm{TOP}} \\ +1, & u \in S_{\text {ВОT }}\end{cases}
$$

Conditional on $\gamma_{\mathrm{SE}}$ the configuration $\sigma$ on $\bar{S} \backslash U$ is given by the Ising model on $S_{\mathrm{TOP}} \backslash U$ and $S_{\text {вот }} \backslash U$ with minus and plus boundary conditions respectively. Let $\theta$ denote the ensemble of contours of this configuration given by the SW (not SE!) splitting rule. Since the boundary conditions are all minus and all plus, there are no open contours. Every maximal connected segment of $\gamma_{\mathrm{SW}} \backslash \gamma_{\mathrm{SE}}$ must be a subset of one of the closed contours of $\theta$ and must share a common vertex with $\gamma_{\mathrm{SE}}$.

By Theorem 5.3 we see that $\gamma_{\mathrm{SE}} \cup \gamma_{\mathrm{SW}} \subset \Lambda^{*}$ where $\Lambda=\{1, \ldots, \ell\} \times\{-\ell, \ldots, \ell\}$ except with probability $O(\exp (-c \ell))$ for some $c(\beta)>0$. By Claim 5.5 there are at most $2 C_{1}^{\star} m \log m$ vertices in $\mathcal{I}=([a-m, a+m] \times \mathbb{Z}) \cap \gamma_{\mathrm{SE}}$ except with probability $C_{1}^{\star} / m$. For $z, z^{\prime} \in \Lambda^{*}$ the probability that both lie in the same closed contour of $\theta$ is at most 
$\exp \left(-\tau_{\beta}\left(z-z^{\prime}\right)\right)$ by Lemma 2.6. Since the surface tension achieves its minimum on the sphere at $\tau_{\beta}(0)>0[6]$, combining the above estimates we have

$$
\begin{aligned}
\pi_{\bar{S}}^{\eta}(\mathcal{B}) & \leq C \exp (-c \ell)+C_{1}^{\star} / m+\sum_{z \in \mathcal{I}} \sum_{z^{\prime}:\left|z-z^{\prime}\right|>C_{2}^{\star} \log m} \exp \left(-\left|z-z^{\prime}\right| \tau_{\beta}(0)\right) \\
& \leq C \exp (-c \ell)+C_{1}^{\star} / m+C m^{1-C_{2}^{\star} \tau_{\beta}(0)} \log ^{3} m .
\end{aligned}
$$

The desired result follows by choosing $C_{2}^{\star}$ sufficiently large.

Finally we show that the contours $\gamma_{\mathrm{SE}}$ and $\gamma_{\mathrm{SW}}$ are unlikely to travel much farther than $h^{2}$ in the $x$-coordinate before attaining height $h$ or $-h$.

Lemma 5.7. Let $\bar{S}=\bar{S}(a, b)$. For any $w>0$ and $0<h \leq \ell$ define the rectangle

$$
\mathcal{R}=\{a-1 / 2-w, \ldots, a-1 / 2+w\} \times\{1 / 2-h, \ldots, h+1 / 2\} .
$$

Let $\mathcal{B}$ denote the event that the contour $\gamma_{\mathrm{SE}}\left(\right.$ resp. $\left.\gamma_{\mathrm{SW}}\right)$ beginning at $(a-1 / 2,1 / 2)$ exits $\mathcal{R}$ and the first point it hits in $\mathcal{R}^{c}$ is in $\{a-1 / 2-w-1, a-1 / 2+w+1\} \times\{1 / 2-$ $h, \ldots, h+1 / 2\}$. There exists a constant $C_{3}^{\star}(\beta)>0$ independent of $w$ and $h$ such that

$$
\pi_{\bar{S}}^{\eta}(\mathcal{B}) \leq C_{3}^{\star} \exp \left(-w /\left(C_{3}^{\star} h^{2}\right)\right) .
$$

Proof. As before let $u=(a+1 / 2,1 / 2)$ and $v=(b-1 / 2,1 / 2)$ denote the endpoints of the open contour $\gamma$. Let $\mathcal{B}_{r}$ and $\mathcal{B}_{l}$ denote the events that the contour exits to the right, and left of $\mathcal{R}$ respectively so that $\mathcal{B}=\mathcal{B}_{l} \cup \mathcal{B}_{r}$. We will examine the case that the contour exits to the right, and the left case will follow similarly. Fix $c(\beta)=\left(3 e C_{\beta}\right)^{2}$ where $C_{\beta}$ is the constant in Lemma 2.1. For large enough $C_{3}^{\star}$ the bound holds trivially when $w \leq 2 c h^{2}$ so assume that $w>2 c h^{2}$. We may define $M(w, h) \in \mathbb{Z}$ such that $0<w /\left(4 c h^{2}\right) \leq M(w, h) \leq w /\left(c h^{2}\right)$ and $\left|c M h^{2}-(b-a)\right| \geq \frac{1}{2}|b-a|$. Define the set of sequences of points

$$
\Xi=\left\{\left(x_{1}, y_{1}\right), \ldots,\left(x_{M}, y_{M}\right) \in \bar{S}^{M}: \forall i, x_{i}=a+1 / 2+i c h^{2},\left|y_{i}\right| \leq h\right\}
$$

and note that if the contour exits $\mathcal{R}$ to the right then it must pass from $u$ through a sequence of points in $\Xi$ in order, and then to $b$. For a sequence $\xi \in \Xi$ we say that $\lambda$ is $\xi$-admissible if it passes through the points in $\xi$ in order and then returns to $b$. Note also that by the construction of $M$ we have $\left|x_{M}-(b-1 / 2)\right| \geq \frac{1}{2}|b-a|$. Taking a union bound over sequences in $\Xi$ we get

$$
\pi_{\bar{S}}^{\eta}\left(\mathcal{B}_{R}\right) \leq\left[\sum_{\xi \in \Xi} \sum_{\substack{\lambda: \delta \lambda=\{u, v\} \\ \xi \text {-admissible }}} q_{\bar{S}^{*}}(\lambda)\right] /\left[\sum_{\lambda: \delta \lambda=\{u, v\}} q_{\bar{S}^{*}}(\lambda)\right] .
$$

In analogy to Corollary 2.5 and (5.22) one has

$$
\sum_{\substack{\lambda: \delta \lambda=\{u, v\} \\ \xi \text {-admissible }}} q_{\Lambda^{*}}(\lambda) \leq \prod_{i=1}^{M+1} \pi_{\Lambda^{*}}^{*}\left(\sigma_{\left(x_{i}, y_{i}\right)} \sigma_{\left(x_{i-1}, y_{i-1}\right)}\right),
$$


where we denote $u=\left(x_{0}, y_{0}\right)$ and $v=\left(x_{M+1}, y_{M+1}\right)$. Therefore, Lemma 2.1 implies that

$$
\begin{aligned}
\sum_{\substack{\lambda: \delta \lambda=\{u, v\} \\
\xi \text {-admissible }}} q_{\bar{S}^{*}}(\lambda) & \leq \frac{C_{\beta}^{M+1} \exp \left(-\sum_{i=1}^{M+1} \tau_{\beta}\left(\left(x_{i}, y_{i}\right)-\left(x_{i-1}, y_{i-1}\right)\right)\right)}{\sqrt{\prod_{i=1}^{M+1}\left|\left(x_{i}, y_{i}\right)-\left(x_{i-1}, y_{i-1}\right)\right|}} \\
& \leq C_{\beta} \frac{\exp \left(-M-\tau_{\beta}(u-v)\right)}{(3 h)^{M} \sqrt{\frac{1}{2}|b-a|}} .
\end{aligned}
$$

Summing over the $(2 h+1)^{M}$ elements of $\Xi$ we have

$$
\sum_{\substack { \xi \in \Xi \\
\begin{subarray}{c}{\lambda: \delta \lambda=\{u, v\} \\
\xi \text {-admissible }{ \xi \in \Xi \\
\begin{subarray} { c } { \lambda : \delta \lambda = \{ u , v \} \\
\xi \text { -admissible } } }\end{subarray}} q_{\bar{S}^{*}}(\lambda) \leq C_{\beta} \frac{\exp \left(-M-\tau_{\beta}(u-v)\right)}{\sqrt{\frac{1}{2}|b-a|}} .
$$

and it follows (recall (2.3)) that there exists a constant $C(\beta)>0$ such that

$$
\pi_{\bar{S}}^{\eta}\left(\mathcal{B}_{R}\right) \leq C e^{-M}
$$

and a similar estimate holds for $\pi_{\bar{S}}^{\eta}\left(\mathcal{B}_{L}\right)$. Recalling that $M \geq w /\left(4 c h^{2}\right)$ and taking a suitably large $C_{3}^{\star}$ now completes the proof.

We now complete the proof of Lemma 5.4. Recalling that $\Gamma=\gamma_{\mathrm{SE}} \cup \gamma_{\mathrm{SW}}$ we define $\Gamma_{\mathrm{TOP}}$ to be the highest path in $\Gamma$ connecting $(a+1 / 2,1 / 2)$ to $(b-1 / 2,1 / 2)$. First observe that $\Gamma_{\text {TOP }}$ is indeed well defined. The collection of dual edges $\Gamma$ partitions $\bar{S}$ into two infinite components and possibly a number of finite components. To construct $\Gamma_{\text {Tор }}$, view the upper infinite component as a subset of $\mathbb{R}^{2}$ by drawing a unit square centered at each of its points. Then $\Gamma_{\text {TOP }}$ is its "horizontal" boundary connecting $(a+1 / 2,1 / 2)$ to $(b-1 / 2,1 / 2)$. Define $\Gamma_{\text {вот }}$ similarly as the lowest path in $\Gamma$. Then

$$
\begin{aligned}
\pi_{\bar{S}}\left(\Gamma_{\text {TOP }} \text { hits } H_{-h}^{*} \text { before hitting } H_{h}^{*} \text { or }(b-1 / 2,1 / 2)\right) \\
\quad \leq \pi_{\bar{S}}\left(\Gamma_{\text {ВОт }} \text { hits } H_{-h}^{*} \text { before hitting } H_{h}^{*} \text { or }(b-1 / 2,1 / 2)\right) \\
\quad=\pi_{\bar{S}}\left(\Gamma_{\text {TOP }} \text { hits } H_{h}^{*} \text { before hitting } H_{-h}^{*} \text { or }(b-1 / 2,1 / 2)\right),
\end{aligned}
$$

where the inequality follows by the fact that $\Gamma_{\text {тор }}$ lies above $\Gamma_{\text {вот }}$ while the equality is by the symmetry of $\Gamma$. It follows that

$$
\pi_{\bar{S}}\left(\Gamma_{\text {TOP }} \text { hits } H_{-h}^{*} \text { before hitting } H_{h}^{*} \text { or }(b-1 / 2,1 / 2)\right) \leq 1 / 2 .
$$

Lemma 5.7 guarantees that except with probability $O\left(\exp \left(-h^{2}\right)\right)$ both contours $\gamma_{\mathrm{SE}}$ and $\gamma_{\mathrm{SW}}$ hit either $H_{-h-C^{\star} \log h}^{*}$ or $H_{h-C^{\star} \log h}^{*}$ before traveling distance order $h^{4}$ in the horizontal direction, and so we only need to consider the interval $\left[a-h^{4}, a+h^{4}\right]$. Now set

$$
C^{\star}:=8 C_{1}^{\star}\left(\beta, C_{2}^{\star}\right)+4 C_{2}^{\star}(\beta)
$$


where $C_{1}^{\star}, C_{2}^{\star}$ are the constants from Claims 5.5 and 5.6. This guarantees that

$$
\begin{aligned}
\operatorname{gn}\left(\gamma_{\mathrm{SE}},\left[a-h^{4}, a+h^{4}\right], 4 C_{2}^{\star} \log h\right) & \leq 4 C_{1}^{\star}\left(\beta, 4 C_{2}^{\star}\right) \log h, \\
\operatorname{gn}\left(\gamma_{\mathrm{SW}},\left[a-h^{4}, a+h^{4}\right], 4 C_{2}^{\star} \log h\right) & \leq 4 C_{1}^{\star}\left(\beta, 4 C_{2}^{\star}\right) \log h, \\
\max _{\substack{(x, y) \in \gamma_{\mathrm{SW}} \\
x \in\left[a-h^{4}, a+h^{4}\right]}} d\left((x, y), \gamma_{\mathrm{SE}}\right) & \leq 4 C_{2}^{\star} \log h,
\end{aligned}
$$

and similarly around $b$ with probability at least $1-C^{\star} / h$. In particular, given the above event we conclude that the vertical distance between $\Gamma_{\mathrm{TOP}}$ and $\gamma_{\mathrm{SE}}$ does not exceed $C^{\star} \log h$ in the intervals $\left[a-h^{4}, a+h^{4}\right]$ and $\left[b-h^{4}, b+h^{4}\right]$, which together with (5.23) completes the proof.

Proof of Theorem 5.1, lower bound. The proof proceeds by progressively revealing the contour $\gamma_{\mathrm{SE}}$. Let $w_{0}$ denote some large constant and let $w_{i}=2 w_{i-1}-2 C^{\star} \log w_{i-1}$ where $C^{\star}$ is the constant from Lemma 5.4. Taking $w_{0}$ sufficiently large we can easily confirm that $w_{j} \geq c 2^{j}$ for some constant $c>0$.

Starting from the left at $(1 / 2,1 / 2)$ for $j \geq 0$ let $A_{l, j}$ be the event that $\gamma_{\mathrm{SE}}$ hits $H_{w_{j}}^{*}$ before hitting $H_{-1}^{*}$ or reaching $(\ell+1 / 2,1 / 2)$. Similarly starting from the right at $(\ell+$ $1 / 2,1 / 2)$ let $A_{r, j}$ be the event that the contour hits $H_{w_{j}}^{*}$ before hitting $H_{-1}^{*}$ or reaching $(1 / 2,1 / 2)$. Let $A_{j}=A_{l, j} \cap A_{r, j}$ and let $B_{j}$ be the event that the contour $\gamma_{\mathrm{SE}}$ hits neither $H_{-1}^{*}$ nor $H_{w_{j}}^{*}$.

We begin by giving a crude lower bound on the probability of $A_{0}$. Let $\mathcal{U}$ be the event that the spin configuration takes the value $(+)$ for all the vertices $(1, j)$ and $(\ell, j)$ for $0 \leq j \leq w_{0}+1$. This occurs with probability at least

$$
\pi_{S}(\mathcal{U}) \geq\left(\frac{1}{2} e^{-8 \beta}\right)^{2 w_{0}+2} .
$$

On the event $\mathcal{U}$ the contour $\gamma_{\mathrm{SE}}(\sigma)$ directly passes from $(1 / 2,1 / 2)$ to $\left(1 / 2, w_{0}+1 / 2\right)$ and from $(\ell+1 / 2,1 / 2)$ to $\left(\ell+1 / 2, w_{0}+1 / 2\right)$. It follows that $\mathcal{U} \subset A_{0}$, and hence

$$
\pi_{S}\left(A_{0}\right) \geq\left(\frac{1}{2} e^{-8 \beta}\right)^{2 w_{0}+2}
$$

so in particular $A_{0}$ occurs with constant probability.

We will establish the following claim.

Claim 5.8. There exists a constant $c>0$ such that for all $j \geq 0$ we have

$$
\pi_{S}\left(A_{j+1} \cup B_{j+1} \mid A_{j}\right) \geq 1 / 4-c 2^{-j} .
$$

If we assume the claim then

$$
\begin{aligned}
\pi_{S}\left(A_{j+1}\right)+\pi_{S}\left(B_{j+1}\right) & =\pi_{S}\left(A_{j+1} \cap A_{j}\right)+\pi_{S}\left(B_{j+1} \cap A_{j}\right)+\pi_{S}\left(B_{j}\right) \\
& \geq\left(1 / 4-c 2^{-j}\right)\left[\pi_{S}\left(A_{j}\right)+\pi_{S}\left(B_{j}\right)\right]
\end{aligned}
$$

where the equality follows from the fact that $A_{j}$ and $B_{j}$ are disjoint. Hence by induction 
and (5.24), for any fixed positive integer $K$,

$$
\begin{aligned}
& P\left(A_{K+\frac{1}{2} \log _{2} \ell}\right)+P\left(B_{K+\frac{1}{2} \log _{2} \ell}\right) \\
& \geq P\left(A_{0}\right) \prod_{j=1}^{K+\frac{1}{2} \log _{2} \ell}\left(1 / 4-c 2^{-j}\right) \geq c^{\prime} 4^{-K} \ell^{-1} .
\end{aligned}
$$

We now prove Claim 5.8. Note that if $\sigma^{\prime} \geq \sigma$ then the curve $\gamma_{\mathrm{SE}}\left(\sigma^{\prime}\right)$ must lie on or above $\gamma_{\mathrm{SE}}(\sigma)$ and hence the event $A_{j+1}$ is increasing in $\sigma$, and so is $A_{j+1} \cup B_{j+1}$. Through a series of monotonicity arguments we will relate this event to that in Lemma 5.4. Suppose that $A_{j}$ holds and that the left part of $\gamma_{\mathrm{SE}}$ first hits $H_{w_{j}}^{*}$ at the dual vertex $\left(z_{j}^{L}+1 / 2, w_{j}+1 / 2\right)$ and denote this part of the contour by $\gamma_{j}^{L}$. Similarly denote by $\gamma_{j}^{R}$ the right part of the contour from $(\ell+1 / 2,1 / 2)$ to $\left(z_{j}^{R}+1 / 2, w_{j}+1 / 2\right)$ with $1 / 2 \leq z_{j}^{L}<z_{j}^{R} \leq \ell+1 / 2$. Finally, let $D_{j+1}$ denote the event that the contour $\gamma_{\mathrm{SE}}$ running between $\left(z_{j}^{L}+1 / 2, w_{j}+1 / 2\right)$ and $\left(z_{j}^{R}+1 / 2, w_{j}+1 / 2\right)$ either hits $H_{w_{j+1}}^{*}$ at both ends before hitting $H_{-1}^{*}$, or hits neither $H_{w_{j+1}}^{*}$ nor $H_{-1}^{*}$. With these definitions we claim that

$$
\pi_{S}\left(A_{j+1} \cup B_{j+1} \mid A_{j}\right) \geq \pi_{S}^{j}\left(D_{j+1} \mid \sigma_{U_{j}}=\eta_{U_{j}}\right),
$$

where $\pi_{S}^{j}$ denote the measure on the strip $S$ with boundary condition given by (+) up to $w_{j}$ and $(-)$ above $w_{j}$,

$$
U_{j}=\left(\left[1, z_{j}^{L}+1\right] \cup\left[z_{j}^{R}, \ell\right]\right) \times\left\{w_{j}, w_{j}+1\right\}
$$

and

$$
\eta_{\left(u_{1}, u_{2}\right)}= \begin{cases}+1, & u_{2}=w_{j} \\ -1, & u_{2}=w_{j}+1\end{cases}
$$

The sequence of monotonicity arguments establishing (5.26) is best explained schematically_-see Fig. 11 and its caption.

Let $D_{j+1}^{L}$ denote the event that the contour $\gamma_{\mathrm{SE}}$ from $\left(z_{j}^{L}+1 / 2, w_{j}+1 / 2\right)$ (resp. $\left.\left(z_{j}^{R}+1 / 2, w_{j}+1 / 2\right)\right)$ hits $H_{w_{j+1}}^{*}$ or $\left(z_{j}^{R}+1 / 2, w_{j}+1 / 2\right)\left(\right.$ resp. $\left.\left(z_{j}^{L}+1 / 2, w_{j}+1 / 2\right)\right)$ before hitting $H_{-1}^{*}$. Clearly $D_{j+1}=D_{j+1}^{L} \cap D_{j+1}^{R}$ and by symmetry we have

$$
\pi_{S}^{j}\left(D_{j+1}^{L} \mid \sigma_{U_{j}}=\eta_{U_{j}}\right)=\pi_{S}^{j}\left(D_{j+1}^{R} \mid \sigma_{U_{j}}=\eta_{U_{j}}\right) .
$$

Since these events are both monotone, by the FKG inequality we have

$$
\pi_{S}^{j}\left(D_{j+1} \mid \sigma_{U_{j}}=\eta_{U_{j}}\right) \geq \pi_{S}^{j}\left(D_{j+1}^{L} \mid \sigma_{U_{j}}=\eta_{U_{j}}\right)^{2} .
$$

It finally suffices to note that the conditional event $D_{j+1}^{L}$ is exactly the event considered in Lemma 5.4 once we shift the strip down by $w_{j}$ and set $h=w_{j}-C^{\star} \log w_{j}, a=z_{j}^{L}$ and $b=z_{j}^{R}-1$. Then we have

$$
\pi_{S}^{j}\left(D_{j+1}^{L} \mid \sigma_{U_{j}}=\eta_{U_{j}}\right) \geq 1 / 2-O\left(1 / w_{j}\right) \geq 1 / 2-c 2^{-j},
$$

which completes the proof of Claim 5.8. 


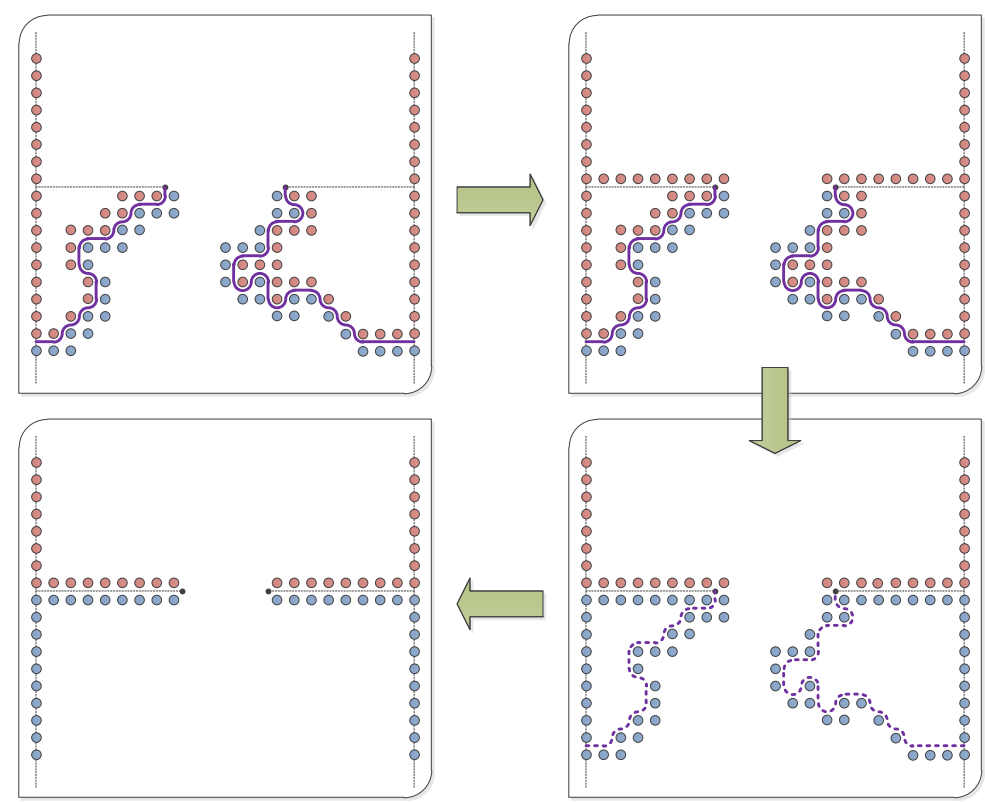

Fig. 11. Monotonicity transformations to reduce the two segments of the open contour in the infinite strip to the setting of Lemma 5.4. First (top-right) impose extra (-) spins at height $w_{j}+1$, making the increasing event $A_{j+1} \cup B_{j+1}$ more unlikely. Next (bottom-right) set the spins at height $w_{j}$ and above the contours $\gamma_{j}^{L}, \gamma_{j}^{R}$ to (+); this does not change the probability of $A_{j+1} \cup B_{j+1}$ due to the Markov property of the Gibbs measure. Finally (bottom-left) remove the constraint that the spins just below $\gamma_{j}^{L}, \gamma_{j}^{R}$ are (+), again making the event $A_{j+1} \cup B_{j+1}$ more unlikely. The dotted line is at height $w_{j}+1 / 2$.

To complete the proof of the lower bound in Theorem 5.1 we will show that

$$
P\left(B_{K+1+\frac{1}{2} \log _{2} \ell} \mid A_{K+\frac{1}{2} \log _{2} \ell}\right)>1 / 2
$$

for a large enough constant $K=K(\beta)$. Applying the same monotonicity transformations as in Claim 5.8 this reduces to the probability of a contour reaching height $w_{j+1}-w_{j}>$ $c 2^{K} \sqrt{\ell}$, which is less than $1 / 2$ for large enough $K$ by Theorem 5.3. Combining with (5.25) implies that

$$
P\left(B_{K+1+\frac{1}{2} \log _{2} \ell}\right)>c \ell^{-1},
$$

as required.

Proof of Theorem 5.1, upper bound. Let $\mathbb{H}=\{w=(x, y): y \geq 0\}$ denote the upper half-plane and consider the correlation between the spins at $u=(1 / 2,1 / 2)$ and $v=(\ell+1 / 2,1 / 2)$ in $\mathbb{H}^{*}$. It is known (see e.g. [38, p. 161, Eq. (5.29)]) that for some $C_{1}(\beta)>0$,

$$
\pi_{\mathbb{H}^{*}}^{*}\left(\sigma_{u} \sigma_{v}\right) \leq \frac{C_{1}}{\ell^{3 / 2}} e^{-\tau_{\beta}(u-v)}
$$


By GKS we can reduce the domain to the dual half-strip of width $|u-v|$,

$$
S^{+}=\mathbb{H}^{*} \cap\{w=(x, y): 1 / 2 \leq x \leq \ell+1 / 2\},
$$

and obtain

$$
\pi_{\mathbb{H}^{*}}^{*}\left(\sigma_{u} \sigma_{v}\right) \geq \pi_{S^{+}}^{*}\left(\sigma_{u} \sigma_{v}\right)=\sum_{\substack{\gamma \in S^{+} \\ \delta \gamma=\{u, v\}}} q_{S^{+}}(\gamma) \geq \sum_{\substack{\gamma \in S^{+} \\ \delta \gamma=\{u, v\}}} q_{S}(\gamma),
$$

where $S=\left\{w=(x, y) \in \mathbb{Z}_{2}^{*}: 1 / 2 \leq x \leq \ell+1 / 2\right\}$ is the dual strip and the last inequality is justified by Lemma 2.2. Combining with (2.3) we can conclude that

$$
\pi_{S}^{\eta}\left(\sigma: \lambda(\sigma) \text { stays above } H_{-1}^{*}\right)=\frac{\sum_{\substack{\gamma \in S^{+} \\ \delta \gamma=\{u, v\}}} q_{S}(\gamma)}{\sum_{\substack{\gamma \in S \\ \delta \gamma=\{u, v\}}} q_{S}(\gamma)} \leq \frac{C_{1} \ell^{-3 / 2} e^{-\tau_{\beta}(u-v)}}{c_{2} \ell^{-1 / 2} e^{-\tau_{\beta}(u-v)}}=C / \ell,
$$

thus completing the proof.

Proof of Corollary 5.2. The upper bound follows directly from the GKS inequalities and the exact solution [38] in the infinite half-plane. As for the lower bound, one has

$$
\begin{aligned}
\pi_{S^{+}}^{*}\left(\sigma_{u} \sigma_{v}\right) & =\sum_{\lambda \in S^{+}: \delta \lambda=\{u, v\}} q_{S^{+}}(\lambda) \geq \sum_{\lambda \in S^{+}: \delta \lambda=\{u, v\}} q_{S^{*}}(\lambda) \\
& =\frac{\sum_{\lambda \in S^{+}: \delta \lambda=\{u, v\}} q_{S^{*}}(\lambda)}{\sum_{\lambda \in S^{*}: \delta \lambda=\{u, v\}} q_{S^{*}}(\lambda)} \sum_{\lambda \in S^{*}: \delta \lambda=\{u, v\}} q_{S^{*}}(\lambda) \geq \frac{c}{\ell} \pi_{S^{*}}^{*}\left(\sigma_{u} \sigma_{v}\right)
\end{aligned}
$$

where in the first inequality we applied Lemma 2.2 and in the second one we used (2.7) and the lower bound of Theorem 5.1. Recalling (2.3), we obtain the desired result.

\section{Proofs of Propositions 4.4 and 4.5}

The remaining part of this work is devoted to the proof of the two equilibrium estimates needed for the new recursive scheme (detailed in Section 4) using the estimates obtained thus far.

\subsection{Proof of Proposition 4.4}

Let $u, v$ be the initial and final point of $\lambda$ and as before let $H_{h}^{*}$ denote level $h$ of the rectangle $R^{*}$. By $(2.8)$ we have

$$
\pi^{(-,-,+,-)}(\sigma: \lambda(\sigma) \text { reaches height } \delta \alpha \sqrt{\ell})
$$


Observe that by monotonicity, (6.1) is increasing in the height of $R$ and so without loss of generality we take

$$
R=S^{+}=\left\{\left(x_{1}, x_{2}\right): 1 \leq x_{1} \leq \ell \text { and } x_{2} \geq 1\right\} .
$$

We now bound separately the numerator and the denominator in (6.1). By the same argument used to prove (5.9) (note that here we bound the probability of the contour exceeding height $\delta \alpha \sqrt{\ell}<\ell$ by the assumptions on $\delta$ and $\alpha$ ), there exists some $c_{1}(\beta)>0$ such that for any $z \in H_{\delta \alpha \sqrt{\ell}}^{*}$,

$$
\sum_{\substack{\lambda: \delta \lambda=\{u, v\} \\ z \in \lambda}} q_{R^{*}}(\lambda) \leq \frac{c_{1}}{\ell} \exp \left(-\tau_{\beta}(0) \ell-\kappa_{\beta}(\delta \alpha)^{2}\right),
$$

and therefore

$$
\sum_{z \in H_{\delta \alpha \sqrt{\ell}}^{*}} \sum_{\substack{\lambda: \delta \lambda=\{u, v\} \\ z \in \lambda}} q_{R^{*}}(\lambda) \leq c_{1} \exp \left(-\tau_{\beta}(0) \ell-\kappa_{\beta}(\delta \alpha)^{2}\right) .
$$

Next we bound from below the denominator in (6.1). Recall that $S$ is the infinite strip $S=\left\{\left(x_{1}, x_{2}\right): 1 \leq x_{1} \leq \ell\right\}$. Then by Lemma 2.2,

$$
\sum_{\substack{\lambda \subset R_{n}^{*} \\ \delta \lambda=\{u, v\}}} q_{R^{*}}(\lambda) \geq\left[\frac{\sum_{\lambda \subset R^{*}, \delta \lambda=\{u, v\}} q_{S^{*}}(\lambda)}{\sum_{\lambda \subset S^{*}, \delta \lambda=\{u, v\}} q_{S^{*}}(\lambda)}\right] \sum_{\substack{\lambda \subset S^{*} \\ \delta \lambda=\{u, v\}}} q_{S^{*}}(\lambda) .
$$

The last factor is estimated in (2.3) while the first factor can be interpreted as the probability in the canonical ensemble given by the weights $q_{S^{*}}(\cdot)$ and conditioned to start at $u$ and to end at $v$ that the contour $\lambda$ stays above the line at height $-1 / 2$. By Theorem 5.1, this is of order $c / \ell$. The desired claim then immediately follows.

\subsection{Proof of Proposition 4.5}

Let $R$ be the $\ell \times \alpha \sqrt{\ell}$ rectangle given by the endpoints $\left(x, y, y^{\prime}, x^{\prime}\right)$ clockwise starting from the Northwest corner, and let $u$ and $v$ denote the West and East endpoints of the interval $\Delta$ centered on the South border, as was shown in Figure 7 in $\S 4$. Recall that in our setting we have a b.c. $\eta$ which is $(-)$ in the North and on $\Delta$ and $(+)$ otherwise, and that the event $\mathcal{V}$ states that $\Delta$ is connected to the North via two contours confined to the left and right halves of $R$ respectively.

Our first step in establishing that $\mathcal{V}$ occurs except with probability $c_{1} \ell^{c_{1}} \exp \left(-c_{2} \alpha^{2}\right)$ is eliminating (except with the aforementioned error probability) the scenario where open contours connect $x$ to $y$ and $u$ to $v$.

Lemma 6.1. Let $R=\left(x, y, y^{\prime}, x^{\prime}\right)$ and $\Delta$ be an interval of length $s \alpha^{2}$ centered on the South border $x^{\prime} y^{\prime}$. For any $\beta>\beta_{c}$ there exist $c_{3}, c_{4}>0$ and $s_{0}>0$ depending only on $\beta$ such that if $s \geq s_{0}$ then for any $\ell \in \mathbb{N}$ we have

$$
\pi_{R}^{\eta}\left(\delta \lambda_{1}=\{x, y\}, \delta \lambda_{2}=\{u, v\}\right) \leq \ell^{c_{3}} \exp \left(-c_{4} \alpha^{2}\right) .
$$


Proof. Our starting point is the equality (2.8), which allows us to rewrite the probability of certain contours in terms of their weights:

$$
\pi_{R}^{\eta}\left(\delta \lambda_{1}=\{x, y\}, \delta \lambda_{2}=\{u, v\}\right)=\Psi_{1} / \Psi_{2}
$$

where

$$
\Psi_{1}:=\sum_{\substack{\lambda=\lambda_{1} \sqcup \lambda_{2} \\ \delta \lambda_{1}=\{\bar{x}, y\}, \delta \lambda_{2}=\{u, v\}}} q_{R^{*}}(\underline{\lambda}), \quad \Psi_{2}:=\sum_{\substack{\lambda=\lambda_{1} \sqcup \lambda_{2} \\ \delta \underline{\lambda}=\{x, y, u, v\}}} q_{R^{*}}(\underline{\lambda}) .
$$

By Lemma 2.4 we have

$$
\Psi_{1} \leq \sum_{\substack{\lambda_{1} \\ \delta \lambda_{1}=\{x, y\}}} q_{R^{*}}\left(\lambda_{1}\right) \sum_{\substack{\lambda_{2} \\ \delta \lambda_{2}=\{u, v\}}} q_{R^{*}}\left(\lambda_{2}\right)=\pi_{R^{*}}^{*}\left(\sigma_{x} \sigma_{y}\right) \pi_{R^{*}}^{*}\left(\sigma_{u} \sigma_{v}\right),
$$

where the last equality is by (2.7). Plugging in Lemma 2.1 it now follows that

$$
\Psi_{1} \leq \exp \left(-\left[\tau_{\beta}(x-y)+\tau_{\beta}(u-v)\right]\right)=\exp \left(-\left[\ell+s \alpha^{2}\right] \tau_{\beta}(0)\right)
$$

Next we consider $\Psi_{2}$. As before, $(2.7)$, the GKS-inequalities $([21,26])$ and symmetry imply that

$$
\Psi_{2}=\pi_{R^{*}}^{*}\left(\sigma_{x} \sigma_{y} \sigma_{u} \sigma_{v}\right) \geq \pi_{R^{*}}^{*}\left(\sigma_{x} \sigma_{u}\right) \pi_{R^{*}}^{*}\left(\sigma_{y} \sigma_{v}\right)=\pi_{R^{*}}^{*}\left(\sigma_{x} \sigma_{u}\right)^{2}
$$

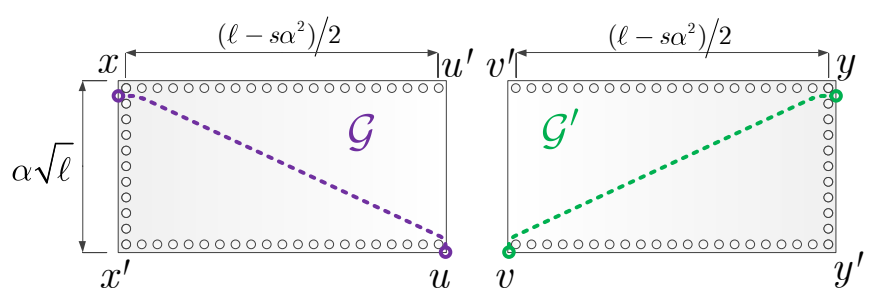

Fig. 12. Domain decrease from the rectangle $R$ to a disjoint union of rectangles separating the two open contours.

Furthermore, by GKS, spin-spin correlations are non-decreasing in the domain so we can clearly confine our domain to the disjoint union of the two rectangles $\mathcal{G}=\left(x, u^{\prime}, u, x^{\prime}\right)$ as shown in Figure 12, and obtain, by symmetry,

$$
\Psi_{2} \geq X^{2} \quad \text { where } X:=\pi_{\mathcal{G}^{*}}^{*}\left(\sigma_{x} \sigma_{u}\right) .
$$

To control the value of $X$, let $z$ be the center of the rectangle $\mathcal{G}$ and further define $\mathcal{G}_{1}=$ $\left(x, a, b, x^{\prime}\right)$ and $\mathcal{G}_{2}=\left(a, u^{\prime}, u, b\right)$ to be the left and right halves of $\mathcal{G}$, each of dimensions $\frac{1}{4}\left(\ell-s \alpha^{2}\right) \times \alpha \sqrt{\ell}$. See Figure 13 for an illustration. The GKS-inequalities (together with a reduction of the domain) yield

$$
\pi_{\mathcal{G}^{*}}^{*}\left(\sigma_{x} \sigma_{u}\right) \geq \pi_{\mathcal{G}_{1}^{*}}^{*}\left(\sigma_{x} \sigma_{z}\right) \pi_{\mathcal{G}_{2}^{*}}^{*}\left(\sigma_{u} \sigma_{z}\right) .
$$




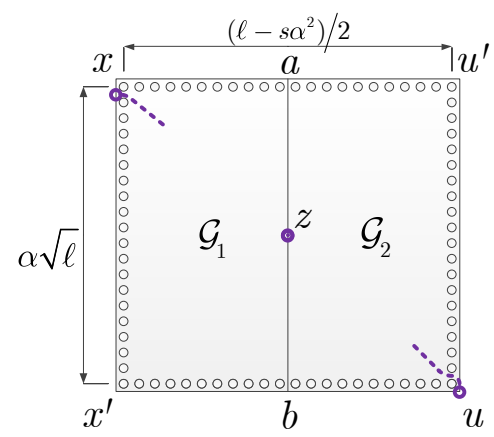

Fig. 13. Comparing the weight of contours from $x$ to $u$ to that of contours connecting both points to the center of the rectangle $z$.

Another application of (2.7) gives

$$
X \geq \sum_{\substack{\lambda \subset \mathcal{G}_{1} \\ \delta \lambda=\{x, z\}}} q_{\mathcal{G}_{1}^{*}}(\lambda) \sum_{\substack{\lambda \subset \mathcal{G}_{2} \\ \delta \lambda=\{u, z\}}} q_{\mathcal{G}_{2}^{*}}(\lambda)=\left(\sum_{\substack{\lambda \subset \mathcal{G}_{1} \\ \delta \lambda=\{x, z\}}} q_{\mathcal{G}_{1}^{*}}(\lambda)\right)^{2},
$$

with the equality due to symmetry. Define $\bar{S}$ to be the infinite half-strip of width $\frac{1}{4}\left(\ell-s \alpha^{2}\right)$ obtained by extending the South border of $\mathcal{G}_{1}$ (i.e. the edge $b x^{\prime}$ ) to $-\infty$. Since $\mathcal{G}_{1}$ is a subgraph of $\bar{S}$ it follows from Lemma 2.2 that

$$
X \geq \bar{X}^{2} \quad \text { where } \quad \bar{X}:=\sum_{\substack{\lambda \subset \mathcal{G}_{1} \\ \delta \lambda=\{x, z\}}} q_{\bar{S}^{*}}(\lambda)
$$

We now claim that

$$
\bar{X} / \bar{Y} \geq 1-(\ell / 4)^{c_{1}} \exp \left(-c_{2} \alpha^{2}\right)
$$

where

$$
\bar{Y}:=\sum_{\substack{\lambda \subset \bar{S} \\ \delta \lambda=\{x, z\}}} q_{\bar{S}^{*}}(\lambda)
$$

Indeed, $\bar{X} / \bar{Y}$ is precisely the probability that the contour $\lambda \subset \bar{S}_{n}$ whose endpoints are $\delta \lambda=\{x, z\}$ stays above the horizontal line $x^{\prime} b$ (the South border of $\mathcal{G}_{1}$ ). If $z^{\prime}$ is the midpoint of $x$ and $x^{\prime}$ then by monotonicity if we condition all $(+)$ in the rectangle $\left(x, a, z, z^{\prime}\right)$ then this only increases the probability that the contour hits the line $x^{\prime} b$. Proposition 4.4 then establishes (6.6). Taking $c_{3}$ large enough in the statement of Lemma 6.1 we can assume that $\alpha$ is at least a large constant times $\sqrt{\log \ell}$, in which case (6.6) gives $\bar{X}=(1-o(1)) \bar{Y}$.

On the other hand, taking $S$ to be the strip obtained by extending the North and South boundaries of $\mathcal{G}_{1}$ to $\pm \infty$, we can now express $\bar{Y}$ in terms of

$$
Y:=\sum_{\substack{\lambda \subset S \\ \delta \lambda=\{x, z\}}} q_{S^{*}}(\lambda)
$$


To this end, observe that $\bar{Y} / Y$ is the probability that the open contour $\lambda$ connecting $x, z$ in $S$ stays below the horizontal line $x a$ (the North border of $\mathcal{G}_{1}$ ). Therefore, by monotonicity and Theorem 5.1 there exists some $c=c(\beta)$ such that

$$
\bar{Y} / Y \geq c / \ell
$$

and on the other hand [20, formula (2.22)] gives, for some $c_{\beta}>0$,

$$
Y=\frac{c_{\beta}+o(1)}{\sqrt{\ell}} \exp \left(-\tau_{\beta}(x-z)\right) .
$$

Combining (6.6)-(6.8) it now follows that

$$
\bar{X} \geq c_{1}^{\prime} \ell^{-c_{2}^{\prime}} \exp \left(-\tau_{\beta}(x-z)\right),
$$

and recalling that $\Psi_{2} \geq X^{2} \geq \bar{X}^{4}$ we deduce that there exists some $c^{\prime \prime}(\beta)>0$ such that

$$
\Psi_{2} \geq c^{\prime \prime} \ell^{-c^{\prime \prime}} \exp \left(-2 \tau_{\beta}(x-u)\right) .
$$

To conclude the proof, we combine (6.5), (6.9) to get

$$
\Psi_{1} / \Psi_{2} \leq\left(1 / c^{\prime \prime}\right) \ell^{c^{\prime \prime}} \exp \left(-\left[\ell+s \alpha^{2}\right] \tau_{\beta}(0)+2 \tau_{\beta}(x-u)\right) .
$$

Recall that $\tau_{\beta}(\theta)$ is an analytic and even function of $\theta$ for any $\beta>\beta_{c}$, hence in particular there exists some $c=c(\beta)>0$ such that

$$
\tau_{\beta}(\theta)-\tau_{\beta}(0) \leq c \theta^{2} \quad \text { for any } \theta \in \mathbb{R} .
$$

Since in our case $\theta \leq \arctan \left(\frac{\alpha \sqrt{\ell}}{\ell / 2}\right) \leq 2 \alpha / \sqrt{\ell}$ it follows that for some $c^{\prime}=c^{\prime}(\beta)>0$,

$$
\tau_{\beta}(x-u) \leq \tau_{\beta}(0)|x-u|+c^{\prime} \alpha^{2} .
$$

On the other hand,

$$
\ell+s \alpha^{2}-|x-u|=O\left(s \alpha^{2}\right)
$$

Combining these inequalities completes the proof.

We next wish to show that whenever $\delta \lambda_{1}=\{x, u\}, \delta \lambda_{2}=\{y, v\}$, the corresponding open contours are also confined to $R_{l}$ and $R_{r}$, the left and right halves of $R$ respectively, except with an appropriate exponentially small probability. The complement event we wish to analyze is illustrated in Figure 14.

Lemma 6.2. Let $R=\left(x, y, y^{\prime}, x^{\prime}\right)$ and $\Delta$ be an interval of length $s \alpha^{2}$ centered on the South border $x^{\prime} y^{\prime}$. Denote by $R_{l}$ and $R_{r}$ the left and right halves of $R$ respectively. For any $\beta>\beta_{c}$ there exist $c_{5}, c_{6}>0$ and $s_{0}>0$ depending only on $\beta$ such that if $s \geq s_{0}$ then for any $\ell \in \mathbb{N}$ we have

$$
\pi_{R}^{\eta}\left(\lambda_{1} \subset R_{l}, \lambda_{2} \subset R_{r} \mid \delta \lambda_{1}=\{x, u\}, \delta \lambda_{2}=\{y, v\}\right) \geq 1-\ell^{c_{5}} \exp \left(-c_{6} \alpha^{2}\right) .
$$




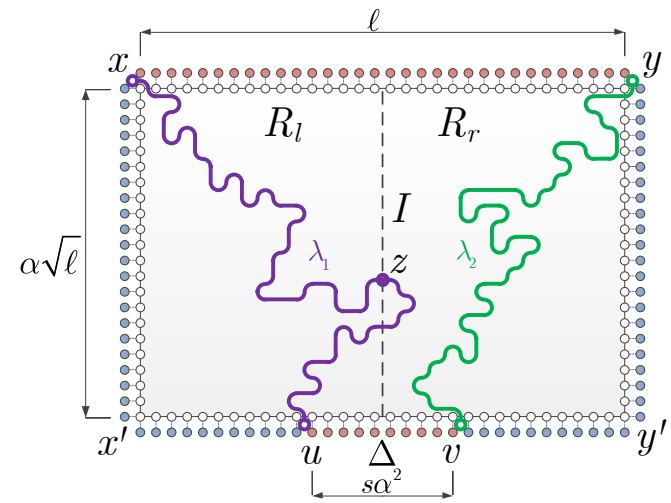

Fig. 14. Open contours under b.c. $(-,+, \Delta)$ crossing the central column of $R$, addressed by the estimate in Lemma 6.2.

Proof. Let $I$ denote the central column of $R$, i.e. $I$ is the vertical line connecting the centers of the North and South boundaries of $R$. We aim to bound the probability that the contour connecting $x$ to $u$ crosses $I$, and similarly for the contour connecting $y$ to $v$. By (2.8) we can write the former probability as $\Phi_{1} / \Phi_{2}$ where

$$
\Phi_{1}:=\sum_{\substack{\lambda=\left(\lambda_{1}, \lambda_{2}\right) \\ \delta \lambda_{1}=\{x, u\}, \lambda_{1} \cap I \neq \emptyset \\ \delta \lambda_{2}=\{y, v\}}} q_{R^{*}}(\underline{\lambda}), \quad \Phi_{2}:=\sum_{\substack{\lambda=\left(\lambda_{1}, \lambda_{2}\right) \\ \delta \underline{\lambda}_{1}=\{x, u\} \\ \delta \underline{\lambda}_{2}=\{y, v\}}} q_{R^{*}}(\underline{\lambda}),
$$

and a union bound (together with symmetry) gives

$$
\pi_{R}^{\eta}\left(\lambda_{1} \subset R_{l}, \lambda_{2} \subset R_{r} \mid \delta \lambda_{1}=\{x, y\}, \delta \lambda_{2}=\{u, v\}\right) \geq 1-2 \frac{\Phi_{1}}{\Phi_{2}} .
$$

To bound $\Phi_{2}$ from below we compare it to $\Psi_{1}, \Psi_{2}$ defined in (6.4). Indeed,

$$
\frac{\Phi_{2}}{\Psi_{2}}=1-\frac{\Psi_{1}}{\Psi_{2}}=1-\pi_{R}^{\eta}\left(\delta \lambda_{1}=\{x, y\}, \delta \lambda_{2}=\{u, v\}\right) \geq 1-\ell^{c_{3}} \exp \left(-c_{4} \alpha^{2}\right),
$$

where the last inequality is precisely the statement of Lemma 6.1. Combining this with the estimate on $\Psi_{2}$ given in (6.9) we conclude that for some absolute $c^{\prime \prime}>0$,

$$
\Phi_{2} \geq\left(1-\ell^{c_{3}} \exp \left(-c_{4} \alpha^{2}\right)\right) c^{\prime \prime} \ell^{-c^{\prime \prime}} e^{-\tau_{\beta}(x-u)-\tau_{\beta}(y-v)}
$$

(we can assume that $\alpha$ is at least a large constant times $\sqrt{\log \ell}$, otherwise the statement of Lemma 6.2 trivially holds).

Remark. The estimate given in (6.9) for $\Psi_{2}$ in the proof of Lemma 6.1 in fact had an exponent of $-2 \tau_{\beta}(x-u)$. The above bound featuring $-\left(\tau_{\beta}(x-u)+\tau_{\beta}(y-v)\right)$ in the exponent readily follows from (6.9) by the symmetry between $\{x, u\}$ and $\{y, v\}$ in the definition of $\Psi_{2}$. 
It remains to bound $\Phi_{1}$. To this end, for a given contour $\gamma$ define $\mathcal{G}_{\gamma}$ to be the graph with the edge-set $R^{*} \backslash \Delta(\gamma)$, where $\Delta(\gamma)$ is the edge-boundary of the contour $\gamma$. Crucially, in our case the edge-boundary of $\gamma$ is disjoint from the edges of $\lambda_{1}$ since these contours are compatible. Therefore, by Lemma 2.3,

$$
q_{R^{*}}(\lambda, \gamma)=q_{R^{*}}(\gamma) q_{\mathcal{G}_{\gamma}}(\lambda)
$$

and plugging this into the definition of $\Phi_{1}$ we deduce that

$$
\Phi_{1}=\sum_{\gamma: \delta \gamma=\{y, v\}}\left(q_{R^{*}}(\gamma) \sum_{\substack{\lambda: \delta \lambda=\{x, u\} \\ \lambda \cap I \neq \emptyset}} q_{\mathcal{G}_{\gamma}}(\lambda)\right) .
$$

The sum over the weights $q_{\mathcal{G}_{\gamma}}$ can be estimated via Corollary 2.5 using a simple union bound (see e.g. [11, Eq. (3.3)] for a similar argument). Indeed,

$$
\sum_{\substack{\lambda: \delta \lambda=\{x, u\} \\ \lambda \cap I \neq \emptyset}} q_{\mathcal{G}_{\gamma}}(\lambda) \leq \sum_{z \in I} \sum_{\substack{\lambda: \delta \lambda=\{x, u\} \\ z \in \lambda}} q_{\mathcal{G}_{\gamma}}(\lambda) \leq \sum_{z \in I} \pi_{\mathcal{G}_{\gamma}}^{*}\left(\sigma_{x} \sigma_{z}\right) \pi_{\mathcal{G}_{\gamma}}^{*}\left(\sigma_{u} \sigma_{z}\right) .
$$

At the same time, we can use (2.7) and then increase the appropriate domains to the infinite-volume lattice to obtain

$$
\begin{aligned}
& \sum_{\substack{\lambda: \delta \gamma=\{y, v\} \\
\lambda: \delta \lambda=\{x, u\} \\
\lambda \cap I \neq \emptyset}} q_{R^{*}}(\gamma) \leq \pi_{\infty}^{*}\left(\sigma_{y} \sigma_{v}\right), \\
& \sum_{\substack{\lambda \in I\\
}} \pi_{\infty}^{*}\left(\sigma_{x} \sigma_{z}\right) \pi_{\infty}^{*}\left(\sigma_{u} \sigma_{z}\right),
\end{aligned}
$$

where $\pi_{\infty}^{*}$ denotes the (unique) Gibbs measure on $\mathbb{Z}^{2}$ at $\beta^{*}$. Altogether,

$$
\begin{aligned}
\Phi_{1} & \leq \sum_{z \in I} \pi_{\infty}^{*}\left(\sigma_{x} \sigma_{z}\right) \pi_{\infty}^{*}\left(\sigma_{z} \sigma_{u}\right) \pi_{\infty}^{*}\left(\sigma_{y} \sigma_{v}\right) \\
& \leq \sum_{z \in I} \exp \left(-\tau_{\beta}(z-x)-\tau_{\beta}(z-u)-\tau_{\beta}(y-v)\right) .
\end{aligned}
$$

At the same time, due to the sharp triangle inequality property of the surface tension ((2.2)), for any $\beta>\beta_{c}$ there exists some $\kappa_{\beta}>0$ such that for any $u, x, z$,

$$
\tau_{\beta}(z-x)+\tau_{\beta}(u-z) \geq \tau_{\beta}(u-x)+\kappa_{\beta}(|z-x|+|u-z|-|u-x|) .
$$

Combining the last two displays implies that

$$
\Phi_{1} \leq e^{-\tau_{\beta}(x-u)-\tau_{\beta}(y-v)} \sum_{z \in I} \exp \left[-\kappa_{\beta}(|z-x|+|u-z|-|u-x|)\right] .
$$

The first term above cancels when combining (6.11), (6.10) and we find that for some $c^{\prime \prime}>0$

$$
\Phi_{1} / \Phi_{2} \leq c^{\prime \prime} \ell^{c^{\prime \prime}} \sum_{z \in I} \exp \left[-\kappa_{\beta}(|z-x|+|u-z|-|u-x|)\right] .
$$


A straightforward manipulation of the above exponent will now complete the proof: One can easily infer from the triangle inequality and symmetry that

$$
\min _{z \in I}(|z-x|+|u-z|-|u-x|)=|v-x|-|u-x|
$$

and recalling that the dimensions of $R$ are $\alpha \sqrt{\ell} \times \ell$ and $|u-v|=s \alpha^{2}$, we have

$$
|v-x|^{2}=\frac{1}{4}\left(\ell+s \alpha^{2}\right)^{2}+\alpha^{2} \ell, \quad|u-x|^{2}=\frac{1}{4}\left(\ell-s \alpha^{2}\right)^{2}+\alpha^{2} \ell .
$$

Hence, as long as $\alpha<(1 / s) \sqrt{\ell}$ (guaranteed by the assumptions of Proposition 4.5) we have $|u-x|+|v-x| \leq 2 \ell$ and

$$
|v-x|-|u-x|=\frac{s \alpha^{2} \ell}{|u-x|+|v-x|} \geq \frac{1}{2} s \alpha^{2} .
$$

Plugging this in (6.12) while summing over the $|I|$ values that $z$ can assume gives

$$
\Phi_{1} / \Phi_{2} \leq c^{\prime \prime} \ell^{c^{\prime \prime}+1} \exp \left(-\left(\kappa_{\beta} / 2\right) s \alpha^{2}\right)
$$

(with room to spare), as required.

Acknowledgments. We are very grateful to Yvan Velenik for valuable suggestions on the randomline representation and related equilibrium estimates, as well as for pointing out the novelty of Corollary 5.2. EL and AS are grateful to the Mathematics Department of the University of Roma Tre for hospitality and support. FM and FLT are similarly grateful to the Theory Group of Microsoft Research. FLT acknowledges partial support by ANR grant LHMSHE.

This work was supported by the European Research Council through the "Advanced Grant" PTRELSS 228032.

\section{References}

[1] Abraham, D. B., Reed, P.: Phase separation in the two-dimensional Ising ferromagnet. Phys. Rev. Lett. 33, 377-379 (1974)

[2] Alexander, K. S.: The spectral gap of the 2-D stochastic Ising model with nearly single-spin boundary conditions. J. Statist. Phys. 104, 59-87 (2001) Zbl 0990.82016 MR 1851384

[3] Alexander, K. S., Yoshida, N.: The spectral gap of the 2-D stochastic Ising model with mixed boundary conditions. J. Statist. Phys. 104, 89-109 (2001) Zbl 0990.82015 MR 1851385

[4] Bianchi, A.: Glauber dynamics on nonamenable graphs: boundary conditions and mixing time. Electron. J. Probab. 13, 1980-2012 (2008) Zbl 1187.82073 MR 2453553

[5] Bodineau, T., Martinelli, F.: Some new results on the kinetic Ising model in a pure phase. J. Statist. Phys. 109, 207-235 (2002) Zbl 1027.82028 MR 1927919

[6] Bricmont, J., El Mellouki, A., Fröhlich, J.: Random surfaces in statistical mechanics: roughening, rounding, wetting,.... J. Statist. Phys. 42, 743-798 (1986) MR 0833220

[7] Campanino, M., Ioffe, D., Velenik, Y.: Ornstein-Zernike theory for finite range Ising models above $T_{c}$. Probab. Theory Related Fields 125, 305-349 (2003) Zbl 1032.60093 MR 1964456

[8] Campanino, M., Ioffe, D., Velenik, Y.: Fluctuation theory of connectivities for subcritical random cluster models. Ann. Probab. 36, 1287-1321 (2008) Zbl 1160.60026 MR 2435850 
[9] Caputo, P., Martinelli, F., Simenhaus, F., Toninelli, F. L.: "Zero" temperature stochastic 3D Ising model and dimer covering fluctuations: a first step towards interface mean curvature motion. Comm. Pure Appl. Math. 64, 778-831 (2011) Zbl 1219.82112 MR 2663712

[10] Caputo, P., Martinelli, F., Toninelli, F. L.: Mixing times of monotone surfaces and SOS interfaces: a mean curvature approach. Comm. Math. Phys. 311, 157-189 (2012) Zbl pre06021972 MR 2892467

[11] Cesi, F., Guadagni, G., Martinelli, F., Schonmann, R. H.: On the two-dimensional stochastic Ising model in the phase coexistence region near the critical point. J. Statist. Phys. 85, 55-102 (1996) Zbl 0937.82004 MR 1413237

[12] Chayes, L., Schonmann, R. H., Swindle, G.: Lifshitz' law for the volume of a twodimensional droplet at zero temperature. J. Statist. Phys. 79, 821-831 (1995) Zbl 1081.82544 MR 1330364

[13] Dobrushin, R., Hryniv, O.: Fluctuations of the phase boundary in the 2D Ising ferromagnet. Comm. Math. Phys. 189, 395-445 (1997) Zbl 0888.60083 MR 1480026

[14] Dobrushin, R., Kotecký, R., Shlosman, S.: Wulff Construction. A Global Shape from Local Interaction. Transl. Math. Monogr. 104, Amer. Math. Soc., Providence, RI (1992) Zbl 0917.60103 MR 1181197

[15] Fisher, D. S., Huse, D. A.: Dynamics of droplet fluctuations in pure and random Ising systems. Phys. Rev. B 35, 6841-6846 (1987)

[16] Fontes, L. R., Schonmann, R. H., Sidoravicius, V.: Stretched exponential fixation in stochastic Ising models at zero temperature. Comm. Math. Phys. 228, 495-518 (2002) Zbl 1004.82013 MR 1918786

[17] Fortuin, C. M., Kasteleyn, P. W., Ginibre, J.: Correlation inequalities on some partially ordered sets. Comm. Math. Phys. 22, 89-103 (1971) Zbl 0346.06011 MR 0309498

[18] Gallavotti, G.: The phase separation line in the two-dimensional Ising model. Comm. Math. Phys. 27, 103-136 (1972) MR 0342116

[19] Glauber, R. J.: Time-dependent statistics of the Ising model. J. Math. Phys. 4, 294-307 (1963) Zbl 0145.24003 MR 0148410

[20] Greenberg, L., Ioffe, D.: On an invariance principle for phase separation lines. Ann. Inst. Henri Poincaré Probab. Statist. 41, 871-885 (2005) Zbl 1115.60039 MR 2165255

[21] Griffiths, R. B.: Correlations in Ising ferromagnets. III. A mean-field bound for binary correlations. Comm. Math. Phys. 6, 121-127 (1967) MR 1552524

[22] Higuchi, Y.: On some limit theorems related to the phase separation line in the twodimensional Ising model. Z. Wahrsch. Verw. Gebiete 50, 287-315 (1979) Zbl 0406.60084 MR 0554548

[23] Hryniv, O.: On local behaviour of the phase separation line in the 2D Ising model. Probab. Theory Related Fields 110, 91-107 (1998) Zbl 0897.60038 MR 1602044

[24] Ioffe, D.: Large deviations for the 2D Ising model: a lower bound without cluster expansions. J. Statist. Phys. 74, 411-432 (1994) Zbl 0946.82502 MR 1257822

[25] Ioffe, D.: Exact large deviation bounds up to $T_{c}$ for the Ising model in two dimensions. Probab. Theory Related Fields 102, 313-330 (1995) Zbl 0830.60018 MR 1339736

[26] Kelly, D. G., Sherman, S.: General Griffiths' inequalities on correlations in Ising ferromagnets. J. Math. Phys. 9, 466-484 (1968)

[27] Levin, D. A., Peres, Y., Wilmer, E.: Markov Chains and Mixing Times. Amer. Math. Soc. (2009) Zbl 1160.60001 MR 2466937

[28] Lifshitz, I. M.: Kinetics of ordering during second-order phase transitions. Soviet J. Experiment. Theor. Phys. 15, 939 (1962)

[29] Liggett, T. M.: Interacting Particle Systems. Classics in Math., Springer, Berlin (2005) Zbl 1103.82016 MR 2108619 
[30] Lubetzky, E., Sly, A.: Critical Ising on the square lattice mixes in polynomial time. Comm. Math. Phys. 313, 815-836 (2012) Zbl pre06065318 MR 2945623

[31] Martinelli, F.: Lectures on Glauber dynamics for discrete spin models. In: Lectures on Probability Theory and Statistics (Saint-Flour, 1997), Lecture Notes in Math. 1717, Springer, Berlin, 93-191 (1999) Zbl 1051.82514 MR 1746301

[32] Martinelli, F.: On the two-dimensional dynamical Ising model in the phase coexistence region. J. Statist. Phys. 76, 1179-1246 (1994) Zbl 0839.60087 MR 1298100

[33] Martinelli, F., Olivieri, E.: Approach to equilibrium of Glauber dynamics in the one phase region. I. The attractive case. Comm. Math. Phys. 161, 447-486 (1994) Zbl 0793.60110 MR 1269387

[34] Martinelli, F., Olivieri, E.: Approach to equilibrium of Glauber dynamics in the one phase region. II. The general case. Comm. Math. Phys. 161, 487-514 (1994) Zbl 0793.60111 MR 1269388

[35] Martinelli, F., Sinclair, A.: Mixing time for the solid-on-solid model. In: STOC'09-Proc. 2009 ACM International Symposium on Theory of Computing, ACM, 571-580 (2009) MR 2780103

[36] Martinelli, F., Sinclair, A., Weitz, D.: Glauber dynamics on trees: boundary conditions and mixing time. Comm. Math. Phys. 250, 301-334 (2004) Zbl 1076.82010 MR 2094519

[37] Martinelli, F., Toninelli, F. L.: On the mixing time of the 2D stochastic Ising model with "plus" boundary conditions at low temperature. Comm. Math. Phys. 296, 175-213 (2010) Zbl 1191.82012 MR 2606632

[38] McCoy, B. M., Wu, T. T.: The Two-Dimensional Ising Model. Harvard Univ. Press, Cambridge, MA (1973) Zbl 1094.82500

[39] Ogielski, A. T.: Dynamics of fluctuations in the ordered phase of kinetic Ising models. Phys. Rev. B 36, 7315-7318 (1987)

[40] Onsager, L.: Crystal statistics. I. A two-dimensional model with an order-disorder transition. Phys. Rev. (2) 65, 117-149 (1944) Zbl 0060.46001 MR 0010315

[41] Peres, Y.: Lectures on "Mixing for Markov Chains and Spin Systems". (Univ. of British Columbia, August 2005); summary at http://www.stat.berkeley.edu/ peres/ubc.pdf

[42] Pfister, C.-E.: Large deviations and phase separation in the two-dimensional Ising model. Helv. Phys. Acta 64, 953-1054 (1991) MR 1149430

[43] Pfister, C.-E., Velenik, Y.: Large deviations and continuum limit in the 2D Ising model. Probab. Theory Related Fields 109, 435-506 (1997) Zbl 0904.60022 MR 1483597

[44] Pfister, C.-E., Velenik, Y.: Interface, surface tension and reentrant pinning transition in the 2D Ising model. Comm. Math. Phys. 204, 269-312 (1999) Zbl 0937.82016 MR 1704276

[45] Saloff-Coste, L.: Lectures on finite Markov chains. In: Lectures on Probability Theory and Statistics (Saint-Flour, 1996), Lecture Notes in Math. 1665, Springer, Berlin, 301-413 (1997) Zbl 0885.60061 MR 1490046

[46] Shlosman, S. B.: The droplet in the tube: a case of phase transition in the canonical ensemble. Comm. Math. Phys. 125, 81-90 (1989) Zbl 0679.60099 MR 1017740

[47] Spohn, H.: Interface motion in models with stochastic dynamics. J. Statist. Phys. 71, 10811132 (1993) Zbl 0935.82546 MR 1226387

[48] Sugimine, N.: A lower bound on the spectral gap of the 3-dimensional stochastic Ising models. J. Math. Kyoto Univ. 42, 751-788 (2003) (2002) Zbl 1163.82303 MR 1967057

[49] Thomas, L. E.: Bound on the mass gap for finite volume stochastic Ising models at low temperature. Comm. Math. Phys. 126, 1-11 (1989) Zbl 0679.60102 MR 1027910

[50] Wilson, D. B.: Mixing times of Lozenge tiling and card shuffling Markov chains. Ann. Appl. Probab. 14, 274-325 (2004) Zbl 1040.60063 MR 2023023 